\title{
Fermion Ito's Formula and Stochastic Evolutions *
}

\author{
D. B. Applebaum ${ }^{\star \star}$ and R. L. Hudson
}

Mathematics Department, University of Nottingham, University Park, Nottingham NG7 2RD, England

\begin{abstract}
An Ito product formula is proved for stochastic integrals against Fermion Brownian motion, and used to construct unitary processes satisfying stochastic differential equations. As in the corresponding Boson theory [10, 11] these give rise to stochastic dilations of completely positive semigroups.
\end{abstract}

\section{Introduction}

In [10] a quantum stochastic calculus leading to an Ito product formula was developed which, in its simplest form, uses as integrators the Boson field operators

$$
A(t)=a\left(\chi_{[0, t]}\right), \quad A^{\dagger}(t)=a^{\dagger}\left(\chi_{[0, t]}\right) .
$$

Here $\chi_{S}$ denotes the indicator function of the set $S$ and the operators (1.1) are the smeared fields corresponding to $\chi_{[0, t]}$ living in the Boson Fock space over the Hilbert space

$$
h=L^{2}[0, \infty) \text {. }
$$

Under the duality transformation this Fock space transforms into the $L^{2}$-space of Wiener measure in such a way that $A(t)+A^{\dagger}(t)$ becomes multiplication by Brownian motion; thus the operators (1.1) constitute a quantum Brownian motion [6]. The Ito product formula can be summarized by the multiplication rules for stochastic differentials

\begin{tabular}{l|lll} 
& $d A$ & $d A^{\dagger}$ & $d t$ \\
\hline$d A$ & 0 & $d t$ & 0 \\
$d A^{\dagger}$ & 0 & 0 & 0 \\
$d t$ & 0 & 0 & 0
\end{tabular}

which contain the classical Ito formula as a special case [10].

* Work completed in part while the first author was supported by an SERC research studentship, and in part while the second author was visiting the Physics Department of the University of Texas at Austin supported by NSF grant PHY 81-07381

** Present address: Dipartimento di Matematica, II Università degli Studi di Roma, Via Orazio Raimondo, I-00173 (La Romanina) Roma, Italy 
Underlying the theory of [10] is the heuristic principle [7] that the eigenproperty of the coherent states $\psi(f), f \in h$,

$$
a(g) \psi(f)=\langle g, f\rangle \psi(f),
$$

can be written in differential form

$$
d A \psi(f)=f(t) d t \psi(f) .
$$

Matrix elements between coherent states of stochastic integrals can thus be expressed as Lebesgue integrals, permitting the extension of stochastic integration from simple integrands to a natural $L^{2}$ class, and leading to the Ito formula (1.3) as a consequence of the Boson commutation relations.

The resulting stochastic calculus was used in [10] to construct unitary processes which generate stochastic dilations of certain uniformly continuous completely positive semigroups, the general case being given in [11].

In this paper we develop the Fermion analog of this Boson theory, in which the stochastic integrators (1.1) are now Fermion field operators in the Fermion Fock space over the Hilbert space (1.2). Though the connection with classical Brownian motion is now only formal, the same Ito product formula (1.3) is obtained, rigorously justifying its heuristic use in [3].

The use of Grassmannian Fermion coherent states is formally attractive [2], but it does not lend itself to rigorous treatment of the theory. Instead of coherent states we use $m$-particle states

$$
\psi_{m}\left(f_{1}, \ldots, f_{m}\right)=a^{\dagger}\left(f_{m}\right) \ldots a^{\dagger}\left(f_{1}\right) \psi_{0}, \quad m \in \mathbb{N}, \quad f_{1}, \ldots, f_{m} \in h,
$$

where $\psi_{0}$ is the Fock vacuum, for which the analog of the formal relation (1.5) is

$$
d A \psi_{m}\left(f_{1}, \ldots, f_{m}\right)=\sum_{j=1}^{m}(-1)^{m-j} f_{j}(t) d t \psi_{m-1}\left(f_{1}, \ldots \bigwedge^{j} \ldots f_{m}\right) .
$$

Once again matrix elements of stochastic integrals are reduced to Lebesgue integrals, and the Ito formula (1.3) now follows from the canonical anticommutation relations (CAR). However, the proofs are more cumbersome than in the Bose case (which could also be developed as in this paper by using $m$-particle rather than coherent states) requiring frequent inductions on the particle number $m$.

We use the theory as in [10] to construct Fermionic unitary processes and related dilations of completely positive semigroups. While the extension to the general uniformly continuous completely positive semigroup is technically simpler than in the Boson case [11] because of the boundedness of Fermion field operators, it will not be given here.

Other aspects of Fermion stochastic calculus, particularly of the analog of the classical Brownian motion $A(t)+A^{\dagger}(t)$, have been extensively developed in [4]. We show that the Ito-Clifford integral of [4] is contained as a special case of our theory.

We say that densely defined operators are mutually adjoint if each is contained in the adjoint of the other. For $1 \leqq k \leqq n, \sum_{j=1}^{n}$ means $\sum_{\substack{j=1 \\ j \neq k}}^{n}$. 


\section{Preliminaries}

Let $h$ be a Hilbert space. The Fermion Fock space over $h$, the Fock representation of the CAR over $h$ and the Fock vacuum vector are characterized to within unitary equivalence as a triple $\left(\mathscr{H}, a, \psi_{0}\right)$ comprising a Hilbert space $\mathscr{H}$, a conjugate-linear map $a$ from $h$ to the algebra $B(\mathscr{H})$ of bounded operators on $\mathscr{H}$ satisfying

$$
\{a(f), a(g)\}=0, \quad\left\{a(f), a^{\dagger}(g)\right\}=\langle f, g\rangle I,
$$

for all $f, g \in h$, and a unit vector $\psi_{0} \in \mathscr{H}$ such that $a(f) \psi_{0}=0$ for all $f \in h$, and the set of vectors

$$
\psi_{m}\left(f_{1}, \ldots, f_{m}\right)=a^{\dagger}\left(f_{m}\right) \ldots a^{\dagger}\left(f_{1}\right) \psi_{0}, \quad m=0,1,2, \ldots, f_{1}, \ldots, f_{m} \in h
$$

is total in $\mathscr{H}$.

For $f=\left(f_{1}, \ldots, f_{m}\right) \in \prod_{j=1}^{m} h$ and $j=1, \ldots, m$ we write $f^{j}=\left(f_{1}, \ldots \bigwedge^{j} \ldots f_{m}\right)$. Then for arbitrary $m \geqq 0, f \in X_{j=1}^{m} h, g \in h$,

$$
a(g) \psi_{m}(f)=\sum_{j=1}^{m}(-1)^{m-j}\left\langle g, f_{j}\right\rangle \psi_{m-1}\left(f^{j}\right) .
$$

We note also that, since $\left\|a^{\dagger}\left(f_{j}\right)\right\|=\left\|f_{j}\right\|$,

$$
\left\|\psi_{m}\left(f_{1}, \ldots, f_{m}\right)\right\| \leqq \prod_{j=1}^{m}\left\|f_{j}\right\| .
$$

We denote by $\mathscr{H}_{+}$and $\mathscr{H}_{-}$, respectively, the closed spans of the vectors (2.2) with $m$ even and odd, respectively, so that thereby $\mathscr{H}$ is a $\mathbb{Z}_{2}$-graded Hilbert space, that is, $\mathscr{H}$ is the internal direct sum of even and odd subspaces $\mathscr{H}_{+}$and $\mathscr{H}_{-}$. Correspondingly, the algebra $B(\mathscr{H})$ is $\mathbb{Z}_{2}$-graded [5] by the rule that $T \in B(\mathscr{H})$ is even if $T \mathscr{H}_{ \pm} \subseteq \mathscr{H}_{ \pm}$and odd if $T \mathscr{H}_{ \pm} \subseteq \mathscr{H}_{\mp}$. The parity of vectors and operators is indicated by the function $\delta$ which is 0 on even elements and 1 on odd.

Now let $h=L^{2}[0, \infty)$. Denote by $h_{t}$ and $h^{t}$ the Hilbert spaces $L^{2}[0, t]$ and $L^{2}(t, \infty)$, by $\mathscr{H}_{t}$ and $\mathscr{H}^{t}$ their respective Fock spaces and by $\psi_{0 t}$ and $\psi_{0}^{t}$ their vacuum vectors. Corresponding to the natural decomposition

$$
h=h_{t} \oplus h^{t}
$$

there is a natural identification [9] $\mathscr{H}=\mathscr{H}_{t} \otimes \mathscr{H}^{t}$ in which

$$
\psi_{0}=\psi_{0 t} \otimes \psi_{0}^{t}
$$

and, for each $f \in h$ having components $f_{t}$ in $h_{t}$ and $f^{t}$ in $h^{t}$,

$$
a(f)=a\left(f_{t}\right) \hat{\otimes} I+I \hat{\otimes} a\left(f^{t}\right) .
$$

Here $\hat{\otimes}$ denotes the anticommuting tensor product of operators defined as follows; if $S \in B\left(\mathscr{H}_{t}\right), T \in B\left(\mathscr{H}^{t}\right), \psi \in \mathscr{H}_{t}$, and $\phi \in \mathscr{H}^{t}$ then, assuming $T$ and $\psi$ have definite parity

$$
S \hat{\otimes} T \psi \otimes \phi=(-1)^{\delta(T) \delta(\psi)} S \psi \otimes T \phi .
$$


We denote by $\mathscr{E}, \mathscr{E}_{+}$, and $\mathscr{E}_{-}$the algebraic spans of the vectors (2.2) in $\mathscr{H}$ with $m$ unrestricted, even and odd, respectively, so that $\mathscr{E}=\mathscr{E}_{+}+\mathscr{E}_{-}$, and by $\mathscr{E}_{t}, \mathscr{E}_{t \pm}, \mathscr{E}^{t}$, and $\mathscr{E}_{ \pm}^{t}$ the counterparts of these subspaces in $\mathscr{H}_{t}$ and $\mathscr{H}^{t}$, respectively. Then for $t \geqq 0$

$$
\mathscr{E}=\mathscr{E}_{t} \otimes \mathscr{E}^{t}, \quad \mathscr{E}_{+}=\mathscr{E}_{t+} \otimes \mathscr{E}_{+}^{t}+\mathscr{E}_{t-} \otimes \mathscr{E}_{-}^{t}, \quad \mathscr{E}_{-}=\mathscr{E}_{t+} \otimes \mathscr{E}_{-}^{t}+\mathscr{E}_{t-} \otimes \mathscr{E}_{+}^{t},
$$

where $\otimes$ denotes the algebraic tensor product.

We are concerned with operator-valued processes which live in the tensor product $h_{0} \otimes \mathscr{H}$ of $\mathscr{H}$ with a Hilbert space $h_{0}$ called the initial space. We write

$$
\tilde{\mathscr{H}}=h_{0} \otimes \mathscr{H}, \quad \tilde{\mathscr{H}}_{t}=h_{0} \otimes \mathscr{H}_{t}, \quad \tilde{\mathscr{E}}=h_{0} \otimes \mathscr{E}, \quad \widetilde{\mathscr{E}}_{t}=h_{0} \otimes \mathscr{E}_{t} .
$$

Then for each $t \geqq 0, \widetilde{\mathscr{H}}=\widetilde{\mathscr{H}}_{t} \otimes \mathscr{H}^{t}, \widetilde{\mathscr{E}}=\widetilde{\mathscr{E}}_{t} \otimes \mathscr{E}^{t}$. We assume that $h_{0}$ is $\mathbb{Z}_{2}$-graded, with even and odd subspaces $h_{0 \pm}$, and we denote by $\theta$ the parity operator, that is the selfadjoint unitary operator which is $I$ on $h_{0+}$ and $-I$ on $h_{0-}$. Then $\widetilde{\mathscr{H}}$ is $\mathbb{Z}_{2}$-graded by

$$
\tilde{\mathscr{H}}_{+}=h_{0+} \otimes \mathscr{H}_{+}+h_{0-} \otimes \mathscr{H}_{-}, \quad \tilde{\mathscr{H}}_{-}=h_{0+} \otimes \mathscr{H}_{-}+h_{0-} \otimes \mathscr{H}_{+} \cdot
$$

We also write

$$
\begin{aligned}
& \widetilde{\mathscr{E}}_{+}=h_{0+} \otimes \mathscr{E}_{+}+h_{0-} \underline{\mathscr{E}}_{-}, \quad \widetilde{\mathscr{E}}_{-}=h_{0+} \otimes \mathscr{E}_{-}+h_{0-} \underline{\otimes} \mathscr{E}_{+}, \\
& \widetilde{\mathscr{E}}_{t+}=h_{0+} \otimes \mathscr{E}_{t+}+h_{0-} \otimes \mathscr{E}_{t-}, \quad \widetilde{\mathscr{E}}_{t-}=h_{0+} \otimes \mathscr{E}_{t-}+h_{0-} \otimes \mathscr{E}_{t+} \cdot
\end{aligned}
$$

An operator $T$ in $\widetilde{\mathscr{H}}_{t}$ with domain $\widetilde{\mathscr{E}}_{t}$ is said to be even if $T \widetilde{\mathscr{E}}_{t \pm} \subseteq \widetilde{\mathscr{H}}_{t \pm}$, and odd if $T \widetilde{\mathscr{E}}_{t \pm} \subseteq \widetilde{\mathscr{H}}_{t \mp}$. Every operator $T$ in $\widetilde{\mathscr{H}}_{t}$ with domain $\widetilde{\mathscr{E}}_{t}$ can then be decomposed uniquely into the sum $T=T_{+}+T_{-}$of even and odd parts.

If $S$ is a bounded operator on $\tilde{\mathscr{H}}^{t}$ its ampliation to $\widetilde{\mathscr{H}}=\widetilde{\mathscr{H}}_{t} \otimes \mathscr{H}^{t}$ is the bounded operator $I \hat{\otimes} S$ on $\widetilde{\mathscr{H}}$. If $T$ is a not necessarily bounded operator in $\tilde{\mathscr{H}}_{t}$ with domain $\widetilde{\mathscr{E}}_{t}$ we define its algebraic ampliation to be the operator in $\mathscr{\mathscr { H }}$ with domain $\widetilde{\mathscr{E}}=\widetilde{\mathscr{E}}_{t-} \otimes \mathscr{H}^{t}$ which acts on product vectors as $T \hat{\otimes} I \psi \otimes \phi=T \psi \otimes \phi\left(\psi \in \widetilde{\mathscr{E}}_{t}, \phi \in \mathscr{H}^{t}\right)$ (note that $I$ is even). If $T$ is of definite parity and $S \in B\left(\mathscr{H}^{t}\right)$ is of definite parity, then as operators on $\widetilde{\mathscr{E}}_{t}-\otimes \mathscr{H}^{t}$,

$$
(T \hat{\otimes} I)(I \hat{\otimes} S)=(-1)^{\delta(T) \delta(S)}(I \hat{\otimes} S)(T \hat{\otimes} I) .
$$

\section{Adapted Processes}

Definition 3.1. By an adapted process we mean a family $F=(F(t): t \geqq 0)$ of operators in $\tilde{\mathscr{H}}$ such that

a) for each $t \geqq 0, F(t)$ is the algebraic ampliation to $\widetilde{\mathscr{E}}_{t} \otimes \mathscr{H}^{t}$ of an operator in $\tilde{\mathscr{H}}_{t}$ with domain $\widetilde{\mathscr{E}}_{t}$;

b) there is a family $F^{\dagger}=\left(F^{\dagger}(t): t \geqq 0\right)$ also satisfying condition a) such that each $F^{\dagger}(t)$ is adjoint to $F(t)$.

Note that in this definition we are guided by [11] rather than by [10]. $F^{\dagger}$ is clearly also an adapted process called the adjoint process to $F$. We denote by $\mathscr{A}$ the complex linear space of all adapted processes. 
Definition 3.2. We say that the adapted process $F$ is simple if there exists an increasing sequence $t_{r}, r=0,1,2, \ldots$ with $t_{0}=0$ and $t_{r} \underset{r}{\longrightarrow} \infty$ such that $F=\sum_{r=0}^{\infty} F_{r} \chi_{\left[t_{r}, t_{r+1}\right)}$, continuous if for arbitrary $u \in h_{0}, m \geqq 0, f_{1}, \ldots, f_{m} \in h$ the vectorvalued functions $t \rightarrow F^{\#}(t) u \otimes \psi_{m}\left(f_{1}, \ldots, f_{m}\right)$, where $F^{\#}$ is either $F$ or $F^{\dagger}$, are strongly continuous on $[0, \infty)$, and locally square integrable if each such function is strongly measurable and satisfies

$$
\int_{0}^{t}\left\|F^{\#}(s) u \otimes \psi_{m}(f)\right\|^{2} d s<\infty
$$

for all $t>0$.

We denote $\mathscr{A}_{0}, \mathscr{A}_{c}$ and $L_{\text {loc }}^{2}$ the subspaces of $\mathscr{A}$ of simple, continuous and locally square integrable processes, respectively. Clearly,

$$
\mathscr{A}_{0}, \mathscr{A}_{c} \subseteq L_{\mathrm{loc}}^{2} .
$$

The following proposition is proved exactly as Proposition 3.2 of [10].

Proposition 3.1. Let $F \in L_{\mathrm{loc}}^{2}$. Then there exists a sequence $F_{n}, n=1,2, \ldots$ of simple processes such that, for each $t>0, u \in h_{0}, m \geqq 0, f_{1}, \ldots, f_{m} \in h$

$$
\lim _{n} \int_{0}^{t}\left\|\left(F^{\#}(s)-F_{n}^{\#}(s)\right) u \otimes \psi_{m}(f)\right\|^{2} d s=0 .
$$

We say that $F \in \mathscr{A}$ is even (respectively odd) if each $F(t)$ is the algebraic ampliation of an even (respectively odd) operator in $\widetilde{\mathscr{H}}_{t}$ with domain $\widetilde{\mathscr{E}}_{t}$. Clearly, every $F \in \mathscr{A}$ is uniquely expressible as the sum $F_{+}+F_{-}$of even and odd parts.

Definition 3.3. The annihilation and creation processes are the mutually adjoint processes $A$ and $A^{\dagger}$ defined by

$$
A(t)=I \hat{\otimes} a\left(\chi_{[0, t]}\right), \quad A^{\dagger}(t)=I \hat{\otimes} a^{\dagger}\left(\chi_{[0, t]}\right), \quad t \geqq 0 .
$$

These processes are clearly odd. Since

$$
\left\|A^{\#}(s)-A^{\#}(t)\right\|^{2}=\left\|I \otimes a^{\#}\left(\chi_{(s, t]}\right)\right\|^{2}=\left\|a^{\#}\left(\chi_{(s, t]}\right)\right\|^{2}=\left\|\chi_{(s, t]}\right\|^{2}=s-t,
$$

it is clear that they are continuous. It follows from (2.1) that for $0 \leqq s<t$ and arbitrary $\phi_{1}, \phi_{2} \in \tilde{\mathscr{H}}$,

$$
\begin{aligned}
& \left\langle\left(A^{\dagger}(t)-A^{\dagger}(s)\right) \phi_{1},\left(A^{\dagger}(t)-A^{\dagger}(s)\right) \phi_{2}\right\rangle \\
& \quad=-\left\langle(A(t)-A(s)) \phi_{1},(A(t)-A(s)) \phi_{2}\right\rangle+(s-t)\left\langle\phi_{1}, \phi_{2}\right\rangle .
\end{aligned}
$$

Let $0 \leqq s<t$ and let $F, F^{\dagger}$ be mutually adjoint operators in $\tilde{\mathscr{H}}$ which are algebraic ampliations to $\widetilde{\mathscr{E}}_{s} \bigotimes \mathscr{H}^{s}$ of operators in $\widetilde{\mathscr{H}}_{s}$ with domain $\widetilde{\mathscr{E}}_{s}$. Since $A_{t}^{\dagger}-A_{s}^{\dagger}$ is bounded and $A_{t}-A_{s}$ maps $\mathscr{E}_{s} \otimes \mathscr{H}^{s}$ to itself, the products $\left(A_{t}^{\dagger}-A_{s}^{\dagger}\right) F$ and $F^{\dagger}\left(A_{t}-A_{s}\right)$ are well defined mutually adjoint operators in $\tilde{\mathscr{H}}$ with domain $\widetilde{\mathscr{E}}_{t} \otimes \mathscr{H}^{t}$, which are algebraic ampliations of operators in $\widetilde{\mathscr{H}}_{t}$ with domain $\widetilde{\mathscr{E}}_{t}$. 


\section{Stochastic Integrals and Ito's Formula for Simple Processes}

Definition 4.1. Let $F, G, H \in \mathscr{A}_{0}$ and suppose that

$$
F=\sum_{r=0}^{\infty} F_{r} \chi_{\left[t_{r}, t_{r+1}\right)}, \quad G=\sum_{r=0}^{\infty} G_{r} \chi_{\left[t_{r}, t_{r+1}\right)}, \quad H=\sum_{r=0}^{\infty} H_{r} \chi_{\left[t_{r}, t_{r+1}\right)},
$$

where

$$
0=t_{0}<t_{1}<\ldots<t_{r} \underset{r}{\longrightarrow} \infty .
$$

Let $M=(M(t): t \geqq 0)$ be the family of operators defined inductively by

$$
\begin{aligned}
M(0)= & 0 ; \\
M(t)= & M\left(t_{r}\right)+\left(A^{\dagger}(t)-A^{\dagger}\left(t_{r}\right)\right) F_{r} \\
& +G_{r}\left(A(t)-A\left(t_{r}\right)\right)+\left(t-t_{r}\right) H_{r}
\end{aligned}
$$

for $t_{r}<t \leqq t_{r+1}$. Then $M$ is called the stochastic integral of $(F, G, H)$ and we write

$$
M(t)=\int_{0}^{t}\left(d A^{\dagger} F+G d A+H d s\right) .
$$

$M$ is an adapted process whose adjoint $M^{\dagger}$ is given by

$$
M^{\dagger}(t)=\int_{0}^{t}\left(d A^{\dagger} G^{\dagger}+F^{\dagger} d A+H^{\dagger} d s\right)
$$

Clearly, $M$ depends linearly on $(F, G, H)$. We use the differential notation $d M$ $=d A^{\dagger} F+G d A+H d t$ to describe the situation that $M=(M(t): t \geqq 0)$ is an adapted process satisfying

$$
M(t)=M(0)+\int_{0}^{t}\left(d A^{\dagger} F+G d A+H d s\right),
$$

where $M(0)$ is the ampliation to $h_{0} \otimes \mathscr{H}$ of an element of $B\left(h_{0}\right)$. Note that, if $d M$ $=d A^{\dagger} F+G d A+H d t$, then

$$
d M_{+}=d A^{\dagger} F_{-}+G_{-} d A+H_{+} d t, \quad d M_{-}=d A^{\dagger} F_{+}+G_{+} d A+H_{-} d t ;
$$

in particular, if $F$ and $G$ are odd and $H$ is even then their stochastic integral is even.

Theorem 4.1. Let $F, G, H \in \mathscr{A}_{0}$ and $M(t)=\int_{0}^{t}\left(d A^{\dagger} F+G d A+H d s\right)$. Then for arbitrary $u, v \in h_{0}, m, n \geqq 0, f_{1}, \ldots, f_{m}, g_{1}, \ldots, g_{n} \in h$ and $t \geqq 0$,

$$
\begin{aligned}
& \left\langle u \otimes \psi_{m}(f), M(t) v \otimes \psi_{n}(g)\right\rangle \\
& =\int_{0}^{t}\left\{\sum_{j=1}^{m}(-1)^{m-j} \overline{f_{j}(s)}\left\langle\theta u \otimes \psi_{m-1}\left(f^{j}\right), F(s) v \otimes \psi_{n}(g)\right\rangle\right. \\
& \quad+\sum_{k=1}^{n}(-1)^{n-k}\left\langle u \otimes \psi_{m}(f), G(s) \theta v \otimes \psi_{n-1}\left(g^{k}\right)\right\rangle g_{k}(s) \\
& \left.\quad+\left\langle u \otimes \psi_{m}(f), H(s) v \otimes \psi_{n}(g)\right\rangle\right\} d s .
\end{aligned}
$$


Proof. Assume $F, G, H$ given by (4.1). Equation (4.5) clearly holds for $t=0$; assume inductively that it holds for $t=t_{r}$. Then for $t_{r}<t \leqq t_{r+1}$ from (4.2)

$$
\begin{aligned}
\langle u \otimes & \left.\psi_{m}(f),\left(M(t)-M\left(t_{r}\right)\right) v \otimes \psi_{n}(g)\right\rangle \\
= & \left\langle u \otimes \psi_{m}(f),\left\{\left(A^{\dagger}(t)-A^{\dagger}\left(t_{r}\right)\right) F_{r}+G_{r}\left(A(t)-A\left(t_{r}\right)\right)+\left(t-t_{r}\right) H_{r}\right\} v \otimes \psi_{n}(g)\right\rangle \\
= & \left\langle\left(A(t)-A\left(t_{r}\right)\right) u \otimes \psi_{m}(f), F_{r} v \otimes \psi_{n}(g)\right\rangle \\
& +\left\langle u \otimes \psi_{m}(f), G_{r}\left(A(t)-A\left(t_{r}\right)\right) v \otimes \psi_{n}(g)\right\rangle \\
& +\left(t-t_{r}\right)\left\langle u \otimes \psi_{m}(f), H_{r} v \otimes \psi_{n}(g)\right\rangle .
\end{aligned}
$$

From (2.8) and (2.3),

Similarly,

$$
\begin{aligned}
\left(A(t)-A\left(t_{r}\right)\right) u \otimes \psi_{m}(f) & =I \hat{\otimes} a\left(\chi_{\left(t_{r}, t\right]}\right) u \otimes \psi_{m}(f)=\theta u \otimes a\left(\chi_{\left(t_{r}, t\right]}\right) \psi_{m}(f) \\
& =\theta u \otimes \sum_{j=1}^{m}(-1)^{m-j}\left\langle\chi_{\left(t_{r}, t\right]}, f_{j}\right\rangle \psi_{m-1}\left(f^{j}\right) \\
& =\sum_{j=1}^{m}(-1)^{m-j} \int_{t_{r}}^{t} f_{j} \theta u \otimes \psi_{m-1}\left(f^{j}\right) .
\end{aligned}
$$

$$
\left(A(t)-A\left(t_{r}\right)\right) v \otimes \psi_{n}(g)=\sum_{k=1}^{n}(-1)^{n-k} \int_{t_{r}}^{t} g_{k} \theta v \otimes \psi_{n-1}\left(g^{k}\right) .
$$

Substituting we find

$$
\begin{aligned}
& \left\langle u \otimes \psi_{m}(f),\left(M(t)-M\left(t_{r}\right)\right) v \otimes \psi_{n}(g)\right\rangle \\
& =\int_{t_{r}}^{t}\left\{\sum_{j=1}^{m}(-1)^{m-j} \overline{f_{j}(s)}\left\langle\theta u \otimes \psi_{m-1}\left(f^{j}\right), F_{r} v \otimes \psi_{n}(g)\right\rangle\right. \\
& \quad+\sum_{k=1}^{n}(-1)^{n-k}\left\langle u \otimes \psi_{m}(f), G_{r} \theta v \otimes \psi_{n-1}\left(g^{k}\right)\right\rangle g_{k}(s) \\
& \left.\quad+\left\langle u \otimes \psi_{m}(f), H_{r} v \otimes \psi_{n}(g)\right\rangle\right\} d s .
\end{aligned}
$$

Since $F(s)=F_{r}, G(s)=G_{r}, H(s)=H_{r}$ for $s \in\left(t_{r}, t\right)$ the result now follows from the additivity of the Lebesgue integral.

Theorem 4.1 implies that the differentials $d A^{\dagger}, d A$, and $d t$ are independent in that, if $d A^{\dagger} F+G d A+H d t=0$, then $F, G$, and $H$ may be equated to zero. Indeed, if the left-hand side of (4.5) vanishes identically in $t$, then, taking (generalized) derivatives of both sides and picking $f$ and $g$ so that each $f_{j}(t)=g_{k}(t)=0$, we see that $H \equiv 0$, since the $\psi_{n}(f)$ and $\psi_{s}(g)$ corresponding to such $f$ and $g$ are total. Relaxing separately the conditions $f_{j}(t)=0, g_{k}(t)=0$ we see similarly that $F=G \equiv 0$.

We shall see that Theorem 4.1 remains true for locally square integrable integrands, as is the following which is then essentially Ito's formula.

Theorem 4.2. Let $F, G, H, F^{\prime}, G^{\prime}, H^{\prime} \in \mathscr{A}_{0}$,

$$
M(t)=\int_{0}^{t}\left(d A^{\dagger} F+G d A+H d s\right), \quad M^{\prime}(t)=\int_{0}^{t}\left(d A^{\dagger} F^{\prime}+G^{\prime} d A+H^{\prime} d s\right) .
$$


Assume that $M$ and $M^{\prime}$ have definite parity. Then for arbitrary $u, v \in h_{0}, m, n \geqq 0$, $f_{1}, \ldots, f_{m}, g_{1}, \ldots, g_{n} \in h$, the function on $(0, \infty), t \mapsto\left\langle M(t) u \otimes \psi_{m}(f), M^{\prime}(t) v \otimes \psi_{n}(g)\right\rangle$, is absolutely continuous with derivative

$$
\begin{aligned}
\frac{d}{d t}\left\langle M(t) u \otimes \psi_{m}(f), M^{\prime}(t) v \otimes \psi_{n}(g)\right\rangle \\
=\sum_{j=1}^{m}(-1)^{m-j} \overline{f_{j}(t)}\left\{\left\langle G(t) \theta u \otimes \psi_{m-1}\left(f^{j}\right), M^{\prime}(t) v \otimes \psi_{n}(g)\right\rangle+(-1)^{\delta(M)}\right. \\
\left.\quad \cdot\left\langle M(t) \theta u \otimes \psi_{m-1}\left(f^{j}\right), F^{\prime}(t) v \otimes \psi_{n}(g)\right\rangle\right\} \\
\quad+\sum_{k=1}^{n}(-1)^{n-k}\left\{(-1)^{\delta\left(M^{\prime}\right)}\left\langle F(t) u \otimes \psi_{m}(f), M^{\prime}(t) \theta v \otimes \psi_{n-1}\left(g^{k}\right)\right\rangle\right. \\
\left.\quad+\left\langle M(t) u \otimes \psi_{m}(f), G^{\prime}(t) \theta v \otimes \psi_{m-1}\left(g^{k}\right)\right\rangle\right\} g_{k}(t) \\
\quad+\left\langle H(t) u \otimes \psi_{m}(f), M^{\prime}(t) v \otimes \psi_{n}(g)\right\rangle+\left\langle M(t) u \otimes \psi_{m}(f), H^{\prime}(t) v \otimes \psi_{n}(g)\right\rangle \\
\quad+\left\langle F(t) u \otimes \psi_{m}(f), F^{\prime}(t) v \otimes \psi_{n}(g)\right\rangle .
\end{aligned}
$$

Proof. Assume $F, G, H$ given by (4.1) and that $F^{\prime}, G^{\prime}, H^{\prime}$ share the same intervals of constancy. Then, for $t \in\left(t_{r}, t_{r+1}\right)$, writing $M(t)$ in the form (4.2) with a similar expression for $M^{\prime}(t)$, we have

where

$$
\left\langle M(t) u \otimes \psi_{m}(f), M^{\prime}(t) v \otimes \psi_{n}(g)\right\rangle=\sum_{i=0}^{3} \sum_{l=0}^{3}\left\langle\phi_{i}, \phi_{l}^{\prime}\right\rangle,
$$

$$
\begin{aligned}
& \phi_{0}=M\left(t_{r}\right) u \otimes \psi_{m}(f), \quad \phi_{1}=\left(A^{\dagger}(t)-A^{\dagger}\left(t_{r}\right)\right) F_{r} u \otimes \psi_{m}(f), \\
& \phi_{2}=G_{r}\left(A(t)-A\left(t_{r}\right)\right) u \otimes \psi_{n}(g), \quad \phi_{3}=\left(t-t_{r}\right) H_{r} u \otimes \psi_{m}(f)
\end{aligned}
$$

with analogous definitions $\phi_{0}^{\prime}, \phi_{1}^{\prime}, \phi_{2}^{\prime}, \phi_{3}^{\prime} .\left\langle\phi_{0}, \phi_{0}^{\prime}\right\rangle$ is independent of $t$ so that

$$
\frac{d}{d t}\left\langle\phi_{0}, \phi_{0}^{\prime}\right\rangle=0 \text {. }
$$

Writing $M\left(t_{r}\right)$ as the algebraic ampliation of an operator in $\tilde{\mathscr{H}}_{t_{r}}$ and using (2.9), (2.8), and (2.3) we have

$$
\begin{aligned}
\left\langle\phi_{0}, \phi_{1}^{\prime}\right\rangle & =\left\langle M\left(t_{r}\right) u \otimes \psi_{m}(f),\left(A^{\dagger}(t)-A^{\dagger}\left(t_{r}\right)\right) F_{r}^{\prime} v \otimes \psi_{n}(g)\right\rangle \\
& =\left\langle\left(A(t)-A\left(t_{r}\right)\right) M\left(t_{r}\right) u \otimes \psi_{m}(f), F_{r}^{\prime} v \otimes \psi_{n}(g)\right\rangle \\
& =(-1)^{\delta(M)}\left\langle M\left(t_{r}\right)\left(I \otimes a\left(\chi_{\left(t_{r}, t\right]}\right)\right) u \otimes \psi_{m}(f), F_{r}^{\prime} v \otimes \psi_{n}(g)\right\rangle \\
& =\sum_{j=1}^{m}(-1)^{\delta(M)+m-j} \int_{t_{r}}^{t} \bar{f}_{j}\left\langle M\left(t_{r}\right) \theta u \otimes \psi_{m-1}\left(f^{j}\right), F_{r}^{\prime} v \otimes \psi_{n}(g)\right\rangle,
\end{aligned}
$$

which is clearly absolute continuous as a function of $t$ with derivative

$$
\begin{aligned}
\frac{d}{d t}\left\langle\phi_{0}, \phi_{1}^{\prime}\right\rangle & =\sum_{j=1}^{m}(-1)^{\delta(M)+m-j} \overline{f_{j}(t)}\left\langle M\left(t_{r}\right) \theta u \otimes \psi_{m-1}\left(f^{j}\right), F_{r}^{\prime} v \otimes \psi_{n}(g)\right\rangle \\
& =\sum_{j=1}^{m}(-1)^{\delta(M)+m-j} \overline{f_{j}(t)}\left\langle M\left(t_{r}\right) \theta u \otimes \psi_{m-1}\left(f^{j}\right), F^{\prime}(t) v \otimes \psi_{n}(g)\right\rangle .
\end{aligned}
$$


Also, from (2.8) and (2.2).

and

$$
\begin{aligned}
\left\langle\phi_{0}, \phi_{2}^{\prime}\right\rangle & =\left\langle\phi_{0}, G_{r}^{\prime}\left(A(t)-A\left(t_{r}\right)\right) v \otimes \psi_{n}(g)\right\rangle \\
& =\sum_{k=1}^{n}(-1)^{n-k}\left\langle\phi_{0}, G_{r}^{\prime} \theta v \otimes \psi_{n-1}\left(g^{k}\right)\right\rangle \int_{t_{r}}^{t} g_{k},
\end{aligned}
$$

so that

$$
\left\langle\phi_{0}, \phi_{3}^{\prime}\right\rangle=\left(t-t_{r}\right)\left\langle\phi_{0}, H_{r}^{\prime} v \otimes \psi_{n}(g)\right\rangle
$$

$$
\begin{aligned}
\frac{d}{d t}\left\langle\phi_{0}, \phi_{2}^{\prime}\right\rangle & =\sum_{k=1}^{n}(-1)^{n-k}\left\langle\varphi_{0}, G_{r}^{\prime} \theta v \otimes \psi_{n-1}\left(g^{k}\right)\right\rangle g_{k}(t) \\
& =\sum_{k=1}^{n}(-1)^{n-k}\left\langle M\left(t_{r}\right) u \otimes \psi_{m}(f), G^{\prime}(t) \theta v \otimes \psi_{n-1}\left(g^{k}\right)\right\rangle g_{k}(t), \\
\frac{d}{d t}\left\langle\phi_{0}, \phi_{3}^{\prime}\right\rangle & =\left\langle\phi_{0}, H_{r}^{\prime} v \otimes \psi_{n}(g)\right\rangle=\left\langle M\left(t_{r}\right) u \otimes \psi_{m}(f), H^{\prime}(t) v \otimes \psi_{n}(g)\right\rangle .
\end{aligned}
$$

By similar arguments to that leading to (4.8) we have

$$
\frac{d}{d t}\left\langle\phi_{1}, \phi_{0}^{\prime}\right\rangle=\sum_{k=1}^{n}(-1)^{\delta\left(M^{\prime}\right)+n-k}\left\langle F(t) u \otimes \psi_{m}(f), M^{\prime}\left(t_{r}\right) \theta v \otimes \psi_{n-1}\left(g^{k}\right)\right\rangle g_{k}(t) .
$$

Using the commutation relation (3.2), (2.9), (2.8), and (2.2) we have

$$
\begin{aligned}
\left\langle\phi_{1},\right. & \left.\phi_{1}^{\prime}\right\rangle=\left\langle\left(A^{\dagger}(t)-A^{\dagger}\left(t_{r}\right)\right) F_{r} u \otimes \psi_{m}(f),\left(A^{\dagger}(t)-A^{\dagger}\left(t_{r}\right)\right) F_{r}^{\prime} v \otimes \psi_{n}(g)\right\rangle \\
= & -\left\langle\left(A(t)-A\left(t_{r}\right)\right) F_{r} u \otimes \psi_{m}(f),\left(A(t)-A\left(t_{r}\right)\right) F_{r}^{\prime} v \otimes \psi_{n}(g)\right\rangle \\
& +\left(t-t_{r}\right)\left\langle F_{r} u \otimes \psi_{m}(f), F_{r}^{\prime} v \otimes \psi_{n}(g)\right\rangle \\
= & -(-1)^{\delta\left(F_{r}\right)+\delta\left(F_{r}^{\prime}\right)}\left\langle F_{r} I \otimes a\left(\chi_{\left(t_{r}, t\right]}\right) u \otimes \psi_{m}(f), F_{r}^{\prime}\left(I \otimes a\left(\chi_{\left(t_{r}, t\right]}\right) v \otimes \psi_{n}(g)\right\rangle\right. \\
& +\left(t-t_{r}\right)\left\langle F_{r} u \otimes \psi_{m}(f), F_{r}^{\prime} v \otimes \psi_{n}(g)\right\rangle \\
= & -(-1)^{\delta\left(F_{r}\right)+\delta\left(F_{r}^{\prime}\right)} \sum_{j=1}^{m}(-1)^{m-j} \sum_{k=1}^{n}(-1)^{n-k} \\
& \cdot \int_{t_{r}}^{t} \bar{f}_{j}\left\langle F_{r} \theta u \otimes \psi_{m-1}\left(f^{j}\right), F_{r}^{\prime} \theta v \otimes \psi_{n-1}\left(g^{k}\right)\right\rangle \\
& \cdot \int_{t_{r}}^{t} g_{k}+\left(t-t_{r}\right)\left\langle F_{r} u \otimes \psi_{m}(f), F_{r}^{\prime} v \otimes \psi_{n}(g)\right\rangle .
\end{aligned}
$$

It follows, using the fact that $F_{r}$ and $M$, and similarly $F_{r}^{\prime}$ and $M^{\prime}$, have opposite parities, that

$$
\begin{aligned}
\frac{d}{d t}\left\langle\phi_{1}, \phi_{1}^{\prime}\right\rangle= & -(-1)^{\delta\left(F_{r}\right)+\delta\left(F_{r}^{\prime}\right)} \sum_{j=1}^{m}(-1)^{m-j} \\
& \cdot \sum_{k=1}^{n}(-1)^{n-k}\left\{\overline{f_{j}(t)} \int_{t_{r}}^{t} g_{k}+\int_{t_{r}}^{t} \overline{f_{j}} g_{k}(t)\right\} \\
& \cdot\left\langle F_{r} \theta u \otimes \psi_{m-1}\left(f^{j}\right), F_{r}^{\prime} \theta v \otimes \psi_{n-1}\left(g^{k}\right)\right\rangle+\left\langle F_{r} u \otimes \psi_{m}(f), F_{r}^{\prime} v \otimes \psi_{n}(g)\right\rangle \\
= & (-1)^{\delta(M)} \sum_{j=1}^{m}(-1)^{m-j} \overline{f_{j}(t)}
\end{aligned}
$$




$$
\begin{aligned}
& \cdot\left\langle F_{r} \theta u \otimes \psi_{m-1}\left(f^{j}\right),\left(A(t)-A\left(t_{r}\right)\right) F_{r}^{\prime} v \otimes \psi_{n}(g)\right\rangle \\
& +(-1)^{\delta\left(M^{\prime}\right)} \sum_{k=1}^{n}(-1)^{n-k} \\
& \left\langle\left(A(t)-A\left(t_{r}\right)\right) F_{r} u \otimes \psi_{m}(f), F_{r}^{\prime} \theta v \otimes \psi_{n-1}\left(g^{k}\right)\right\rangle g_{k}(t) \\
& +\left\langle F_{r} u \otimes \psi_{m}(f), F_{r}^{\prime} u \otimes \psi_{m}(g)\right\rangle \\
= & (-1)^{\delta(M)} \sum_{j=1}^{m}(-1)^{m-j} \overline{f_{j}(t)} \\
& \cdot\left\langle\left(A^{\dagger}(t)-A^{\dagger}\left(t_{r}\right)\right) F_{r} \theta u \otimes \psi_{m-1}\left(f^{j}\right), F^{\prime}(t) v \otimes \psi_{n}(g)\right\rangle \\
& +(-1)^{\delta\left(M^{\prime}\right)} \sum_{k=1}^{n}(-1)^{n-k} \\
& \cdot\left\langle F(t) u \otimes \psi_{m}(f),\left(A^{\dagger}(t)-A^{\dagger}\left(t_{r}\right)\right) F_{r}^{\prime} \theta v \otimes \psi_{n-1}\left(g^{k}\right)\right\rangle g_{k}(t) \\
& +\left\langle F(t) u \otimes \psi_{m}(f), F^{\prime}(t) v \otimes \psi_{n}(g)\right\rangle .
\end{aligned}
$$

The nilpotency relation $a\left(\chi_{\left(t, t_{r}\right]}\right)^{2}=0$ shows that $\left\langle\phi_{1}, \phi_{2}^{\prime}\right\rangle$ vanishes, so that

$$
\frac{d}{d t}\left\langle\phi_{1}, \phi_{2}^{\prime}\right\rangle=0
$$

while, writing $H_{r}^{\prime}$ as the algebraic ampliation of an operator in $\widetilde{\mathscr{H}}_{t_{r}}$ and using (2.9), (2.8), and (2.3)

$$
\begin{aligned}
\left\langle\phi_{1}, \phi_{3}^{\prime}\right\rangle= & (-1)^{\delta\left(H_{r}^{\prime}\right)} \sum_{k=1}^{n}(-1)^{n-k} \\
& \cdot\left\langle F_{r} u \otimes \psi_{m}(f), H_{r}^{\prime} \theta v \otimes \psi_{n-1}\left(g^{k}\right)\right\rangle\left(t-t_{r}\right) \int_{t_{r}}^{t} g_{k},
\end{aligned}
$$

so that, since $H_{r}^{\prime}$ and $M^{\prime}$ have the same parity

$$
\begin{aligned}
\frac{d}{d t}\left\langle\phi_{1}, \phi_{3}^{\prime}\right\rangle= & (-1)^{\delta\left(H_{r}^{\prime}\right)} \sum_{k=1}^{n}(-1)^{n-k}\left\langle F_{r} u \otimes \psi_{m}(f), H_{r}^{\prime} \theta v \otimes \psi_{n-1}\left(g^{k}\right)\right\rangle \\
& \cdot\left\{\int_{t_{r}}^{t} g+\left(t-t_{r}\right) g_{k}(t)\right\} \\
= & \left\langle F_{r} u \otimes \psi_{m}(f),\left(A(t)-A\left(t_{r}\right)\right) H_{r}^{\prime} v \otimes \psi_{n}(g)\right\rangle+(-1)^{\delta\left(M^{\prime}\right)} \sum_{k=1}^{n}(-1)^{n-k} \\
& \cdot\left\langle F_{r} u \otimes \psi_{m}(f),\left(t-t_{r}\right) H_{r}^{\prime} \theta v \otimes \psi_{n-1}\left(g^{k}\right)\right\rangle g_{k}(t) \\
= & \left\langle\left(A^{\dagger}(t)-A^{\dagger}\left(t_{r}\right)\right) F_{r} u \otimes \psi_{m}(f), H^{\prime}(t) v \otimes \psi_{n}(g)\right\rangle+(-1)^{\delta\left(M^{\prime}\right)} \\
& \cdot \sum_{k=1}^{n}(-1)^{n-k}\left\langle F(t) u \otimes \psi_{m}(f),\left(t-t_{r}\right) H_{r}^{\prime} \theta v \otimes \psi_{n-1}\left(g^{k}\right)\right\rangle g_{k}(t)
\end{aligned}
$$

Similar arguments show that

$$
\begin{gathered}
\frac{d}{d t}\left\langle\phi_{2}, \phi_{0}^{\prime}\right\rangle=\sum_{j=1}^{m}(-1)^{m-j} \overline{f_{j}(t)}\left\langle G(t) \theta u \otimes \psi_{m-1}\left(f^{j}\right), M^{\prime}\left(t_{r}\right) v \otimes \psi_{n}(g)\right\rangle, \\
\frac{d}{d t}\left\langle\phi_{2}, \phi_{1}^{\prime}\right\rangle=0,
\end{gathered}
$$




$$
\begin{aligned}
& \frac{d}{d t}\left\langle\phi_{2}, \phi_{2}^{\prime}\right\rangle \\
& =\sum_{j=1}^{m}(-1)^{m-j} \overline{f_{j}(t)}\left\langle G(t) \theta u \otimes \psi_{m-1}\left(f^{j}\right), G_{r}^{\prime}\left(A(t)-A\left(t_{r}\right)\right) v \otimes \psi_{n}(g)\right\rangle \\
& +\sum_{k=1}^{n}(-1)^{n-k}\left\langle G_{r}\left(A(t)-A\left(t_{r}\right)\right) u \otimes \psi_{m}(f), G^{\prime}(t) \theta v \otimes \psi_{n-1}\left(g^{k}\right)\right\rangle g_{k}(t), \\
& \frac{d}{d t}\left\langle\phi_{2}, \phi_{3}^{\prime}\right\rangle=\sum_{j=1}^{m}(-1)^{m-j} \overline{f_{j}(t)}\left\langle G(t) \theta u \otimes \psi_{m-1}\left(f^{j}\right),\left(t-t_{r}\right) H_{r}^{\prime} v \otimes \psi_{n}(g)\right\rangle \\
& +\left\langle G_{r}\left(A(t)-A\left(t_{r}\right)\right) u \otimes \psi_{m}(f), H^{\prime}(t) v \otimes \psi_{n}(g)\right\rangle, \\
& \frac{d}{d t}\left\langle\phi_{3}, \phi_{0}^{\prime}\right\rangle=\left\langle H(t) u \otimes \psi_{m}(f), M^{\prime}\left(t_{r}\right) v \otimes \psi_{n}(g)\right\rangle, \\
& \frac{d}{d t}\left\langle\phi_{3}, \phi_{1}^{\prime}\right\rangle=\left\langle H(t) u \otimes \psi_{m}(f),\left(A^{\dagger}(t)-A^{\dagger}\left(t_{r}\right)\right) F_{r}^{\prime} v \otimes \psi_{n}(g)\right\rangle \\
& +(-1)^{\delta(M)} \sum_{j=1}^{m}(-1)^{m-j} \overline{f_{j}(t)} \\
& \cdot\left\langle\left(t-t_{r}\right) H_{r} \theta u \otimes \psi_{m-1}\left(f^{j}\right), F^{\prime}(t) v \otimes \psi_{n}(g)\right\rangle, \\
& \frac{d}{d t}\left\langle\phi_{3}, \phi_{2}^{\prime}\right\rangle=\sum_{k=1}^{n}(-1)^{n-k}\left\langle\left(t-t_{r}\right) H_{r} u \otimes \psi_{m}(f), G^{\prime}(t) \theta v \otimes \psi_{n-1}\left(g^{k}\right)\right\rangle g_{k}(t) \\
& +\left\langle H(t) u \otimes \psi_{m}(f), G_{r}^{\prime}\left(A(t)-A\left(t_{r}\right)\right) v \otimes \psi_{n}(g)\right\rangle, \\
& \frac{d}{d t}\left\langle\phi_{3}, \phi_{3}^{\prime}\right\rangle=\left\langle\left(t-t_{r}\right) H_{r} u \otimes \psi_{m}(f), H^{\prime}(t) v \otimes \psi_{n}(g)\right\rangle \\
& +\left\langle H(t) u \otimes \psi_{m}(f),\left(t-t_{r}\right) H_{r}^{\prime} v \otimes \psi_{n}(g)\right\rangle .
\end{aligned}
$$

Summing the Eqs. (4.7)-(4.22) and recalling (4.2) and the analogous expression for $M^{\prime}(t)$ establishes (4.6).

\section{Extension to Locally Square Integrable Processes}

Equating $\left(F^{\prime}, G^{\prime}, H^{\prime}\right)$ to $(F, G, H), v$ to $u, n$ to $m$, and $g$ to $f$ in Theorem 4.2 we get

$$
\begin{aligned}
\frac{d}{d t} \| & M(t) u \otimes \psi_{m}(f) \|^{2} \\
= & 2 \operatorname{Re}\left\{\sum _ { j = 1 } ^ { m } ( - 1 ) ^ { m - j } \overline { f _ { j } ( t ) } \left[\left\langle G(t) \theta u \otimes \psi_{m-1}\left(f^{j}\right), M(t) u \otimes \psi_{m}(f)\right\rangle\right.\right. \\
& \left.+(-1)^{\delta(M)}\left\langle M(t) \theta u \otimes \psi_{m-1}\left(f^{j}\right), F(t) u \otimes \psi_{m}(f)\right\rangle\right] \\
& \left.+\left\langle M(t) u \otimes \psi_{m}(f), H(t) u \otimes \psi_{m}(f)\right\rangle\right\}+\left\|F(t) u \otimes \psi_{m}(f)\right\|^{2} \\
\leqq & \sum_{j=1}^{m}\left\{\left|f_{j}(t)\right|^{2}\left[\left\|M(t) \theta u \otimes \psi_{m-1}\left(f^{j}\right)\right\|^{2}+\left\|M(t) u \otimes \psi_{m}(f)\right\|^{2}\right]\right. \\
& \left.+\left\|G(t) \theta u \otimes \psi_{m-1}\left(f^{j}\right)\right\|^{2}\right\}+\left\|M(t) u \otimes \psi_{m}(f)\right\|^{2} \\
& +(m+1)\left\|F(t) u \otimes \psi_{m}(f)\right\|^{2}+\left\|H(t) u \otimes \psi_{m}(f)\right\|^{2}
\end{aligned}
$$


for arbitrary $F, G, H \in \mathscr{A}_{0}$, where we use the inequality $2 \operatorname{Re}\left\langle\phi_{1}, \phi_{2}\right\rangle \leqq\left\|\phi_{1}\right\|^{2}$ $+\left\|\phi_{2}\right\|^{2}$.

Now let $F, G, H \in L_{\text {loc }}^{2}$ By Proposition 3.1 there exist $F_{n}, G_{n}, H_{n} \in \mathscr{A}_{0}$, $n=1,2, \ldots$ such that for arbitrary $u \in h_{0}, m \geqq 0, f_{1}, \ldots, f_{m} \in h$, and $t>0$

$$
\begin{aligned}
& \int_{0}^{t}\left\{\left\|\left(F^{\#}(s)-F_{n}^{\#}(s)\right) u \otimes \psi_{m}(f)\right\|^{2}+\left\|\left(G^{\#}(s)-G_{n}^{\#}(s)\right) u \otimes \psi_{m}(f)\right\|^{2}\right. \\
& \left.+\left\|\left(H^{\#}(s)-H_{n}^{\#}(s)\right) u \otimes \psi_{m}(f)\right\|^{2}\right\} d s \underset{n}{\longrightarrow} 0 .
\end{aligned}
$$

Let

$$
M_{n}(t)=\int_{0}^{t}\left(d A^{\dagger} F_{n}+G_{n} d A+H_{n} d s\right), \quad n=1,2, \ldots, \quad t \geqq 0 .
$$

Theorem 5.1. For arbitrary $u \in h_{0}, m \geqq 0, f_{1}, \ldots, f_{m} \in h$, and $T \geqq 0$ the sequence $M_{n}(t) u \otimes \psi_{m}(f), n=1,2, \ldots$ converges in $\widetilde{\mathscr{H}}$ uniformly for $t \in[0, T]$ to a limit independent of the choice of $F_{n}, G_{n}, H_{n} \in \mathscr{A}_{0}, n=1,2, \ldots$ satisfying (5.2).

Proof. The proof is by induction on $m$. When $m=0$, replacing $M$ by $M_{p}-M_{q}$ in (5.1) gives

$$
\begin{aligned}
\frac{d}{d t}\left\|\left(M_{p}-M_{q}\right) u \otimes \psi_{0}\right\|^{2} \leqq & \left\|\left(M_{p}-M_{q}\right) u \otimes \psi_{0}\right\|^{2}+\left\|\left(F_{p}-F_{q}\right) u \otimes \psi_{0}\right\|^{2} \\
& +\left\|\left(H_{p}-H_{q}\right) u \otimes \psi_{0}\right\|^{2},
\end{aligned}
$$

whence using the integrating factor $e^{-t}$

$$
\begin{aligned}
& \left\|\left(M_{p}(t)-M_{q}(t)\right) u \otimes \psi_{0}\right\|^{2} \\
& \quad \leqq \int_{0}^{t} e^{t-s}\left\{\left\|\left(F_{p}(s)-F_{q}(s)\right) u \otimes \psi_{0}\right\|^{2}+\left\|\left(H_{p}(s)-H_{q}(s)\right) u \otimes \psi_{0}\right\|^{2}\right\} d s \\
& \quad \leqq e^{T} \int_{0}^{T}\left\{\left\|\left(F_{p}(s)-F_{q}(s)\right) u \otimes \psi_{0}\right\|^{2}+\left\|\left(H_{p}(s)-H_{q}(s)\right) u \otimes \psi_{0}\right\|^{2}\right\} d s
\end{aligned}
$$

whenever $t \in[0, T]$. From this it is clear that the sequence $M_{n}(t) u \otimes \psi_{0}, n=1,2, \ldots$ is uniformly Cauchy and thus uniformly convergent for $t \in[0, T]$.

For general $m$, from (5.1)

$$
\begin{aligned}
\frac{d}{d t} \| & \left(M_{p}-M_{q}\right) u \otimes \psi_{m}(f) \|^{2} \\
\leqq & \sum_{j=1}^{m}\left\{\left|f_{j}(t)\right|^{2}\left[\left\|\left(M_{p}-M_{q}\right) \theta u \otimes \psi_{m-1}\left(f^{j}\right)\right\|^{2}+\left\|\left(M_{p}-M_{q}\right) u \otimes \psi_{m}(f)\right\|^{2}\right]\right. \\
& \left.+\left\|\left(G_{p}-G_{q}\right) \theta u \otimes \psi_{m-1}\left(f^{j}\right)\right\|^{2}\right\}+\left\|\left(M_{p}-M_{q}\right) u \otimes \psi_{m}(f)\right\|^{2} \\
& +(m+1)\left\|\left(F_{p}-F_{q}\right) u \otimes \psi_{m}(f)\right\|^{2}+\left\|\left(H_{p}-H_{q}\right) u \otimes \psi_{m}(f)\right\|^{2}
\end{aligned}
$$

Using the integrating factor $\exp \left\{-t-\int_{0}^{t} \sum_{j=1}^{m}\left|f_{j}(s)\right|^{2} d s\right\}$, we find

$$
\begin{aligned}
& \left\|\left(M_{p}(t)-M_{q}(t)\right) u \otimes \psi_{m}(f)\right\|^{2} \\
& \quad \leqq \int_{0}^{t} \exp \left\{\int_{s}^{t}\left(\sum_{j=1}^{m}\left|f_{j}(\tau)\right|^{2}+1\right) d \tau\right\}
\end{aligned}
$$




$$
\begin{aligned}
& \cdot\left[\sum _ { j = 1 } ^ { m } \left\{\left|f_{j}(s)\right|^{2}\left\|\left(M_{p}(s)-M_{q}(s)\right) \theta u \otimes \psi_{m-1}\left(f^{j}\right)\right\|^{2}\right.\right. \\
& \left.+\left\|\left(G_{p}(s)-G_{q}(s)\right) \theta u \otimes \psi_{m-1}\left(f^{j}\right)\right\|^{2}\right\}+(m+1)\left\|\left(F_{p}(s)-F_{q}(s)\right) u \otimes \psi_{m}(f)\right\|^{2} \\
& \left.+\left\|\left(H_{p}(s)-H_{q}(s)\right) u \otimes \psi_{m}(f)\right\|^{2}\right] d s \\
\leqq & \exp \left\{\int_{0}^{T}\left(\sum_{j=1}^{m}\left|f_{j}(\tau)\right|^{2}+1\right) d \tau\right\} \\
& \cdot \int_{0}^{T}\left[\sum _ { j = 1 } ^ { m } \left\{\left|f_{j}(s)\right|^{2}\left\|\left(M_{p}(s)-M_{q}(s)\right) \theta u \otimes \psi_{m-1}\left(f^{j}\right)\right\|^{2}\right.\right. \\
& \left.+\left\|\left(G_{p}(s)-G_{q}(s)\right) \theta u \otimes \psi_{m-1}\left(f^{j}\right)\right\|^{2}\right\}+(m+1)\left\|\left(F_{p}(s)-F_{q}(s)\right) u \otimes \psi_{m}(f)\right\|^{2} \\
& \left.+\left\|\left(H_{p}(s)-H_{q}(s)\right) u \otimes \psi_{m}(f)\right\|^{2}\right] d s .
\end{aligned}
$$

Making the inductive assumption that each $M_{n}(s) \theta u \otimes \psi_{m-1}\left(f^{j}\right), n=1,2, \ldots$ is uniformly convergent hence uniformly Cauchy for $s \in[0, T]$, recalling that each $f_{j}$ is square integrable, and using (5.2) we conclude that $M_{n}(t) \theta u \otimes \psi_{m}(f), n=1,2, \ldots$ is uniformly Cauchy hence uniformly convergent for $t \in[0, T]$. A similar inductive argument shows that the limit does not depend on choice of $\left(F_{n}, G_{n}, H_{n}\right), n=1,2, \ldots$ satisfying (5.2).

The operator $M(t)$ defined on $\widetilde{E}$ by

$$
M(t) u \otimes \psi_{m}(f)=\lim _{n} M_{n}(t) u \otimes \psi_{m}(f)
$$

extends uniquely as an algebraic ampliation to $\widetilde{\mathscr{E}}_{t} \otimes \mathscr{H}^{t}$; we denote the extension also by $M(t)$. Then $M=(M(t): t \geqq 0)$ is an adapted process called the stochastic integral of the locally square integrable processes $(F, G, H)$, and denoted by

$$
M(t)=\int_{0}^{t}\left(d A^{\dagger} F+G d A+H d s\right), \quad t \geqq 0 .
$$

The adjoint process $M^{\dagger}(t)$ is given by

$$
M^{\dagger}(t)=\int_{0}^{t}\left(d A^{\dagger} G^{\dagger}+F^{\dagger} d A+H^{\dagger} d s\right), \quad t \geqq 0 .
$$

In view of the uniformity of the convergence in Theorem 5.1, the integrated form (using the integrating factor $\left.\exp \left\{-\int_{0}^{t}\left(\sum_{j=1}^{m}\left|f_{j}(s)\right|^{2}+1\right) d s\right\}\right)$ of (5.1), namely

$$
\begin{aligned}
& \left\|M(t) u \otimes \psi_{m}(f)\right\|^{2} \\
& \leqq \int_{0}^{t} \exp \left\{\int_{s}^{t}\left(\sum_{j=1}^{m}\left|f_{j}(\tau)\right|^{2}+1\right) d \tau\right\} \\
& \quad \cdot\left[\sum_{j=1}^{m}\left\{\left|f_{j}(s)\right|^{2}\left\|M(s) \theta u \otimes \psi_{m-1}\left(f^{j}\right)\right\|^{2}+\left\|G(s) \theta u \otimes \psi_{m-1}\left(f^{j}\right)\right\|^{2}\right\}\right. \\
& \left.\quad+(m+1)\left\|F(s) u \otimes \psi_{m}(f)\right\|^{2}+\left\|H(s) u \otimes \psi_{m}(f)\right\|^{2}\right] d s
\end{aligned}
$$


remain valid in the transition from simple to locally square integrable integrands, as does the corresponding estimate,

$$
\begin{aligned}
& \left\|(M(t)-M(s)) u \otimes \psi_{m}(f)\right\|^{2} \\
& \left.\leqq \int_{s}^{t} \exp \left(\int_{\tau}^{t} \sum_{j=1}^{m}\left|f_{j}(\sigma)^{2}\right|+1\right) d \sigma\right\} \\
& \cdot\left[\sum_{j=1}^{m}\left\{\left|f_{j}(\tau)\right|^{2}\left\|(M(\tau)-M(s)) \theta u \otimes \psi_{m-1}\left(f^{j}\right)\right\|^{2}+\left\|G(\tau) \theta u \otimes \psi_{m-1}\left(f^{j}\right)\right\|^{2}\right\}\right. \\
& \left.\quad+(m+1)\left\|F(\tau) u \otimes \psi_{m}(f)\right\|^{2}+\left\|H(\tau) u \otimes \psi_{m}(f)\right\|^{2}\right] d \tau,
\end{aligned}
$$

got by replacing $(F, G, H)$ by $\left(F \chi_{[s, \infty)}, G \chi_{[s, \infty)}, H \chi_{[s, \infty)}\right)$ in (5.3). From this it follows that the stochastic integral $M(t)$ is a continuous process; in particular, the functions $t \rightarrow M(t) u \otimes \psi_{m}(f)$ are bounded on finite intervals. From this it follows that we may pass to the limit of simple approximations in (4.5) and the integrated form of (4.6) to obtain that Theorem 4.1 and 4.2 hold for integrands $F, G, H \in L_{\text {loc }}^{2}$.

We denote by $\mathscr{M}$ the set of all adapted processes $M$ satisfying $d M=d A^{\dagger} F$ $+G d A+H d t$ for locally square-integrable $F, G$, and $H$, with the further property that for each $t \geqq 0, M(t), F(t), G(t)$, and $H(t)$ are bounded operators, and

$$
\sup _{0 \leqq s \leqq t} \max \{\|M(s)\|,\|F(s)\|,\|G(s)\|,\|H(s)\|\}<\infty .
$$

Theorem 6.3 below shows that $\mathscr{M}$ is far from empty.

Theorem 5.2. $\mathscr{M}$ is $a^{*}$-algebra under pointwise operator multiplication and the involution $M \rightarrow M^{\dagger}$. Furthermore, for $M_{1}, M_{2} \in \mathscr{M}$,

$$
d\left(M_{1} M_{2}\right)=d M_{1} \cdot M_{2}+M_{1} \cdot d M_{2}+d M_{1} \cdot d M_{2},
$$

where, assuming that $d M_{j}=d A^{\dagger} F_{j}+G_{j} d A+H_{j} d t$ and that $M_{1}, M_{2}$ are of definite parity,

$$
\begin{gathered}
d M_{1} \cdot M_{2}=d A^{\dagger} F_{1} M_{2}+(-1)^{\delta\left(M_{2}\right)} G_{1} M_{2} d A+H_{1} M_{2} d t, \\
M_{1} \cdot d M_{2}=(-1)^{\delta\left(M_{1}\right)} d A^{\dagger} M_{1} F_{2}+M_{1} G_{2} d A+M_{1} H_{2} d t, \\
d M_{1} \cdot d M_{2}=G_{1} F_{2} d t .
\end{gathered}
$$

Proof. The uniform boundedness of $M_{j}, F_{j}, G_{j}$, and $H_{j}$ shows that the integrands on the right-hand side of (5.5) and (5.6) are locally square integrable so that the corresponding stochastic integrals are well defined. Equation (5.4) now follows by combining (4.5) with (4.6), in which we set $M=M_{1}^{\dagger}, M^{\prime}=M_{2}$.

Theorem 5.2 is the rigorous justification of the Ito product formula (1.3) [3].

Just as the classical stochastic integral against Brownian motion is contained as a special case of the Boson stochastic calculus of [10], an Ito-Clifford stochastic integral [4] can be obtained from that developed here as follows.

We take $h_{0}=\mathbb{C}$ so that $\tilde{\mathscr{H}}=\mathscr{H}$. Equipped with the tracial state

$$
\omega(T)=\left\langle\psi_{0}, T \psi_{0}\right\rangle,
$$


the von Neumann algebra $\mathscr{C}$ generated by the operators

$$
\Psi(f)=a(f)+a^{\dagger}(\bar{f}), \quad f \in L^{2}[0, \infty)
$$

is a probability gauge space [12]. The map $T \rightarrow T \psi_{0}$ from $\mathscr{C}$ to $\mathscr{H}$ extends uniquely to a Hilbert space isomorphism $D$, the duality transformation of [13], from $L^{2}(\mathscr{C})$ onto $\mathscr{H} . \mathscr{C}$ is generated by the operators $\Psi(t)=A^{\dagger}(t)+A(t), t \geqq 0$. We denote by $\mathscr{C}_{t}$ the von Neumann subalgebra generated by the $\Psi(s), 0 \leqq s \leqq t$; then $D$ maps $L^{2}\left(\mathscr{C}_{t}\right)$ onto $\mathscr{H}_{t} \otimes \psi_{0}^{t}$, where $\psi_{0}^{t}$ is the vacuum in $\mathscr{H}^{t}$. We write \|\|$_{2}$ for the norm in $L^{2}(\mathscr{C})$ and use the traciality of the state $\omega$ to obtain the estimate

$$
\|G F\|_{2}^{2}=\omega\left(F^{*} G^{*} G F\right)=\omega\left(F F^{*} G^{*} G\right) \leqq\|F\|^{2}\|G\|_{2}^{2},
$$

valid for $F \in \mathscr{C}, G \in L^{2}(\mathscr{C})$.

Theorem 5.3. Let $F:[0, \infty) \rightarrow L^{2}(\mathscr{C})$ be measurable and such that, for each $t \geqq 0$, $\int_{0}^{t}\|F(s)\|_{2}^{2} d s<\infty$ and $F(t) \in L^{2}\left(\mathscr{C}_{t}\right)$, so that [4] the Ito-Clifford integral $\int_{0}^{t} F(s) d \Psi(s)$ exists. Then $F$ is locally square integrable and, denoting its even and odd parts by $F_{ \pm}$ we have

$$
\int_{0}^{t} F(s) d \Psi(s)=\int_{0}^{t}\left(d A^{\dagger}\left(F_{+}-F_{-}\right)+\left(F_{+}+F_{-}\right) d A\right)
$$

as operators with domain $\mathscr{E}_{t} \bigotimes \mathscr{H}^{t}$.

Proof. Suppose first that $F$ is $\mathscr{C}$-valued and simple, say $F=\sum_{r=0}^{\infty} F_{r} \chi_{\left[t_{r}, t_{r+1}\right)}$, where $0=t_{0}<t_{1}<\ldots<t_{r} \underset{r}{\longrightarrow} \infty$. Then [4]

$$
\begin{aligned}
\int_{0}^{t} F(s) d \Psi(s) \\
=\sum_{j=0}^{r} F_{j}\left(\Psi\left(t_{j+1}\right)-\Psi\left(t_{j}\right)\right)+F_{r}\left(\Psi(t)-\psi\left(t_{r}\right)\right), \quad t_{r}<t \leqq t_{r+1} \\
=\sum_{j=0}^{r}\left(F_{j+}+F_{j-}\right)\left(A^{\dagger}\left(t_{j+1}\right)-A^{\dagger}\left(t_{j}\right)+A\left(t_{j+1}\right)-A\left(t_{j}\right)\right) \\
\quad+\left(F_{r+}+F_{r-}\right)\left(A^{\dagger}(t)-A^{\dagger}\left(t_{r}\right)+A(t)-A\left(t_{r}\right)\right) \\
=\sum_{j=0}^{r}\left\{\left(A^{\dagger}\left(t_{j+1}\right)-A^{\dagger}\left(t_{j}\right)\right)\left(F_{j+}-F_{j-}\right)+\left(F_{j+}+F_{j-}\right)\left(A\left(t_{j+1}\right)-A\left(t_{j}\right)\right)\right\} \\
\quad+\left(A^{\dagger}(t)-A^{\dagger}\left(t_{r}\right)\right)\left(F_{j+}-F_{j-}\right)+\left(F_{j+}+F_{j-}\right)\left(A\left(t_{j+1}\right)-A\left(t_{j}\right)\right) \\
=\int_{0}^{t}\left\{d A^{\dagger}\left(F_{+}-F_{-}\right)+\left(F_{+}+F_{-}\right) d A\right\} .
\end{aligned}
$$

More generally, if $F$ satisfies the hypothesis of the theorem there exists a sequence $F_{p}, p=1,2, \ldots$ of simple $\mathscr{C}$-valued processes such that, for each $t \geqq 0$, $\int_{0}^{t}\left\|F(s)-F_{p}(s)\right\|_{2}^{2} d s \underset{p}{\longrightarrow} 0$; then $\int_{0}^{t} F(s) d \Psi(s)=\lim _{p} \int_{0}^{t} F_{p}(s) d \Psi(s)$, where the limit is in 
the norm of $L^{2}\left(\mathscr{C}_{t}\right)$. (In [4] the $F_{p}$ are $L^{2}(\mathscr{C})$-valued, rather than $\mathscr{C}$-valued, however, using the density of each $\mathscr{C}_{t}$ in $L^{2}\left(\mathscr{C}_{t}\right)$ it can be seen that there is no loss of generality in our stronger assumption.) The theorem now follows from the inequality

$$
\left\|G \psi_{m}\left(f_{1} \ldots f_{m}\right)\right\| \leqq P_{m}\|G\|_{2},
$$

where $P_{m}$ is a polynomial in the $\left|\left\langle f_{j}, f_{k}\right\rangle\right|, j, k=1, \ldots, m$ valid for arbitrary $G \in L^{2}(\mathscr{C})$, which will now be proved by induction on $m$. When $m=0,\left\|G \psi_{0}\right\|=\|G\|_{2}$ by definition of \|\|$_{2}$. More generally,

$$
\begin{aligned}
\left\|G \psi_{m}\left(f_{1}, \ldots, f_{m}\right)\right\| & =\left\|G a^{\dagger}\left(f_{m}\right) a^{\dagger}\left(f_{m-1}\right) \ldots a^{\dagger}\left(f_{1}\right) \psi_{0}\right\| \\
& \leqq\left\|G\left(a^{\dagger}\left(f_{m}\right)+a\left(\overline{f_{m}}\right)\right) \psi_{m-1}\left(f^{m}\right)\right\|+\left\|G a\left(\bar{f}_{m}\right) a^{\dagger}\left(f_{m-1}\right) \ldots a^{\dagger}\left(f_{1}\right) \psi_{0}\right\| \\
& \leqq\left\|G\left(a^{\dagger}\left(f_{m}\right)+a\left(\overline{f_{m}}\right)\right) \psi_{m-1}\left(f^{m}\right)\right\|+\sum_{j=1}^{m-1}\left|\left\langle f_{m}, f_{j}\right\rangle\right|\left\|G \psi_{m-2}\left(f^{j, m}\right)\right\| .
\end{aligned}
$$

Inequality (5.9) now follows from the inductive hypothesis, together with the inequality (5.8) in which we take $F=a^{\dagger}\left(f_{m}\right)+a\left(\bar{f}_{m}\right)$.

\section{Stochastic Evolutions}

Let $L_{j}, j=1,2,3$ be bounded operators on the initial space $h_{0}$ and denote by $\tilde{L}_{j}$, $j=1,2,3$ their ampliations to $\tilde{\mathscr{H}}$. Assume $L_{1}, L_{2}$ odd and $L_{3}$ even. Set

$$
C=\max \left\{\left\|L_{j}\right\|, j=1,2,3\right\} .
$$

We show that the stochastic differential equation

$$
d U=d A^{\dagger} U \tilde{L}_{1}+U\left(\tilde{L}_{2} d A+\tilde{L}_{3} d t\right), \quad U(0)=I
$$

has a unique solution.

We establish existence iteratively, defining processes $U_{p}, p=0,1,2, \ldots$ inductively by

$$
\begin{aligned}
& U_{0}(t) \equiv I \\
& U_{p}(t)=I+\int_{0}^{t}\left(d A^{\dagger} U_{p-1} \tilde{L}_{1}+U_{p-1}\left(\tilde{L}_{2} d A+\tilde{L}_{3} d t\right)\right) .
\end{aligned}
$$

Clearly, $U_{0} \in L_{\text {loc }}^{2}$. Assume $U_{p-1} \in L_{\text {loc }}^{2}$. Then the processes $t \rightarrow U_{p-1}(t) \tilde{L}_{j}$ are strongly measurable, moreover, for arbitrary $u \in h_{0}, m \geqq 0, f_{1}, \ldots, f_{m} \in h$, and $t>0$

$$
\begin{aligned}
\int_{0}^{t}\left\|U_{p-1}(s) \tilde{L}_{j} u \otimes \psi_{m}(f)\right\|^{2} d s & =\int_{0}^{t}\left\|U_{p-1}(s) L_{j} u \otimes \psi_{m}(f)\right\| d s, \\
\int_{0}^{t}\left\|\left(U_{p-1}(s) \tilde{L}_{j}\right)^{\dagger} u \otimes \psi_{m}(f)\right\|^{2} d s & =\int_{0}^{t}\left\|\tilde{L}_{j}^{\dagger} U_{p-1}^{\dagger}(s) u \otimes \psi_{m}(f)\right\|^{2} d s \\
& \leqq C^{2} \int_{0}^{t}\left\|U_{p-1}^{\dagger}(s) u \otimes \psi_{m}(f)\right\|^{2} d s,
\end{aligned}
$$

so that these processes are locally square integrable. But then their stochastic integral exists and is likewise locally square integrable. Thus $U_{p}$ is well defined for all $p$. 
Clearly, each $U_{p}$ is even. Also

$$
\begin{aligned}
& U_{0}^{\dagger}(t) \equiv I, \\
& U_{p}^{\dagger}(t)=I+\int_{0}^{t}\left(d A^{\dagger} \tilde{L}_{2}^{\dagger} U_{p-1}^{\dagger}+\tilde{L}_{1}^{\dagger} U_{p-1}^{\dagger} d A+\tilde{L}_{3}^{\dagger} U_{p-1}^{\dagger} d s\right) .
\end{aligned}
$$

Theorem 6.1. For arbitrary $p>0, m \geqq 0, u \in h_{0}, f_{1}, \ldots, f_{m} \in h$, and $t>0$,

$$
\begin{aligned}
& \left\|\left(U_{p}^{\#}(t)-U_{p-1}^{\#}(t)\right) u \otimes \psi_{m}(f)\right\|^{2} \\
& \quad \leqq(p !)^{-1} \exp \left\{t+\int_{0}^{t} \sum_{j=1}^{m}\left|f_{j}\right|^{2}\right\} t^{p} C^{2 p}(2 m+2)^{p+m}\|u\|^{2} \exp \sum_{j=1}^{m}\left\|f_{j}\right\|^{2} .
\end{aligned}
$$

Proof. We give the proof for $U_{p}$, that for $U_{p}^{\dagger}$ being similarly based on the iteration (6.3). The proof is by induction on $m$. From the estimate (5.1) we have

$$
\begin{aligned}
& \frac{d}{d t}\left\|\left(U_{p}-U_{p-1}\right) u \otimes \psi_{m}(f)\right\|^{2} \\
& \leqq \sum_{j=1}^{m}\left\{\left|f_{j}(t)\right|^{2}\left[\left\|\left(U_{p}-U_{p-1}\right) u \otimes \psi_{m}(f)\right\|^{2}+\left\|\left(U_{p}-U_{p-1}\right) \theta u \otimes \psi_{m-1}\left(f^{j}\right)\right\|^{2}\right]\right. \\
& \left.\quad+\left\|\left(U_{p-1}-U_{p-2}\right) L_{2} \theta u \otimes \psi_{m-1}\left(f^{j}\right)\right\|^{2}\right\}+\left\|\left(U_{p}-U_{p-1}\right) u \otimes \psi_{m}(f)\right\|^{2} \\
& \quad+(m+1)\left\|\left(U_{p-1}-U_{p-2}\right) L_{1} u \otimes \psi_{m}(f)\right\|^{2}+\left\|\left(U_{p-1}-U_{p-2}\right) L_{3} u \otimes \psi_{m}(f)\right\|^{2} .
\end{aligned}
$$

Using the integrating factor $\exp \left\{-t-\int_{0}^{t} \sum_{j=1}^{m}\left|f_{j}\right|^{2}\right\}$ we obtain

$$
\begin{aligned}
& \left\|\left(U_{p}(t)-U_{p-1}(t)\right) u \otimes \psi_{m}(f)\right\|^{2} \\
& \leqq \\
& \int_{0}^{t} \exp \left\{t-s+\int_{s}^{t} \sum_{j=1}^{m}\left|f_{j}\right|^{2}\right\} \\
& \quad \cdot\left[\sum_{j=1}^{m}\left|f_{j}(s)\right|^{2}\left\|\left(U_{p}(s)-U_{p-1}(s)\right) \theta u \otimes \psi_{m-1}\left(f^{j}\right)\right\|^{2}\right. \\
& \quad+\sum_{j=1}^{m}\left\|\left(U_{p-1}(s)-U_{p-2}(s)\right) L_{2} \theta u \otimes \psi_{m-1}\left(f^{j}\right)\right\|^{2} \\
& \quad+(m+1)\left\|\left(U_{p-1}(s)-U_{p-2}(s)\right) L_{1} u \otimes \psi_{m}(f)\right\|^{2} \\
& \left.\quad+\left\|\left(U_{p-1}(s)-U_{p-2}(s)\right) L_{3} u \otimes \psi_{m}(f)\right\|^{2}\right] d s .
\end{aligned}
$$

When $m=0,(6.5)$ becomes

$$
\begin{aligned}
& \left\|\left(U_{p}(t)-U_{p-1}(t)\right) u \otimes \psi_{0}\right\|^{2} \\
& \leqq \int_{0}^{t} e^{t-s}\left\{\left\|\left(U_{p-1}(s)-U_{p-2}(s)\right) L_{1} u \otimes \psi_{0}\right\|^{2}\right. \\
& \left.\quad+\left\|\left(U_{p-1}(s)-U_{p-2}(s)\right) L_{3} u \otimes \psi_{0}\right\|^{2}\right\} d s,
\end{aligned}
$$

whence we obtain by repetition that

$$
\left\|\left(U_{p}(t)-U_{p-1}(t)\right) u \otimes \psi_{0}\right\|^{2} \leqq(p !)^{-1} e^{t} t^{p} 2^{p} C^{2 p}\|u\|^{2},
$$


so that (6.4) holds in this case. More generally, making the inductive assumption that

$$
\begin{aligned}
& \left\|\left(U_{p}(s)-U_{p-1}(s)\right) \theta u \otimes \psi_{m-1}\left(f^{j}\right)\right\|^{2} \\
& \quad \leqq(p !)^{-1} \exp \left\{s+\int_{0}^{s} \sum_{k=1}^{m}(j)\left|f_{k}\right|^{2}\right\} s^{p} C^{2 p}(2 m)^{p+m-1}\|u\|^{2} \exp \sum_{k=1}^{m}(j)\left\|f_{k}\right\|^{2},
\end{aligned}
$$

we see that the first term on the right of (6.5) is bounded above by

$$
\begin{aligned}
& \sum_{j=1}^{m} \exp \left\{t+\int_{0}^{t} \sum_{k=1}^{m}\left|f_{k}\right|^{2}\right\} \int_{0}^{t} \exp \left\{\int_{s}^{t}\left|f_{j}\right|^{2}\right\}\left|f_{j}(s)\right|^{2} s^{p} d s(p !)^{-1} C^{2 p} \\
& \cdot(2 m)^{p+m-1}\|u\|^{2} \exp \sum_{k=1}^{m}(j)\left\|f_{k}\right\|^{2} .
\end{aligned}
$$

Since, integrating by parts,

$$
\begin{aligned}
\int_{0}^{t} \exp \left\{\int_{s}^{t}\left|f_{j}\right|^{2}\right\}\left|f_{j}(s)\right|^{2} s^{p} d s & =\int_{0}^{t} \exp \left\{\int_{s}^{t}\left|f_{j}\right|^{2}\right\} p s^{p-1} d s-t^{p} \\
& \leqq \exp \int_{0}^{t}\left|f_{j}\right|^{2} t^{p}
\end{aligned}
$$

this is in turn bounded above by

$$
m \exp \left\{t+\int_{0}^{t} \sum_{j=1}^{m}\left|f_{j}\right|^{2}\right\} t^{p}(p !)^{-1} C^{2 p}(2 m)^{p+m-1}\|u\|^{2} \exp \sum_{j=1}^{m}\left\|f_{j}\right\|^{2} .
$$

Similarly, the second term on the right of $(6.5)$ is bounded above by

$$
\begin{aligned}
& \sum_{j=1}^{m} \exp \left\{t+\int_{0}^{t} \sum_{k=1}^{m}(j)\left|f_{k}\right|^{2}\right\} \int_{0}^{t} \exp \left\{\int_{s}^{t}\left|f_{j}\right|^{2}\right\} s^{p-1} d s((p-1) !)^{-1} C^{2 p-2} \\
& \cdot(2 m)^{p+m-2}\left\|L_{2} \theta u\right\|^{2} \exp \sum_{k=1}^{m}(j)\left\|f_{k}\right\|^{2} \\
& \leqq m \exp \left\{t+\int_{0}^{t} \sum_{j=1}^{m}\left|f_{j}\right|^{2}\right\} t^{p}(p !)^{-1} C^{2 p} \\
& \cdot(2 m)^{p+m-2}\|u\|^{2} \exp \sum_{j=1}^{m}\left\|f_{j}\right\|^{2} .
\end{aligned}
$$

Thus (6.5) gives

$$
\begin{aligned}
& \left\|\left(U_{p}(t)-U_{p-1}(t)\right) u \otimes \psi_{m}(f)\right\|^{2} \\
& \leqq \\
& (p !)^{-1} \exp \left\{t+\int_{0}^{t} \sum_{j=1}^{m}\left|f_{j}\right|^{2}\right\} t^{p} C^{2 p}\|u\|^{2} \exp \left\{\sum_{j=1}^{m}\left\|f_{j}\right\|^{2}\right\} \\
& \quad \cdot m\left((2 m)^{p+m-1}+(2 m)^{p+m-2}\right)+\int_{0}^{t} \exp \left\{t-s+\int_{s}^{t} \sum_{j=1}^{m}\left|f_{j}\right|^{2}\right\} \\
& \cdot\left[(m+1)\left\|\left(U_{p-1}(s)-U_{p-2}(s)\right) L_{1} u \otimes \psi_{m}(f)\right\|^{2}\right. \\
& \left.\quad+\left\|\left(U_{p-1}(s)-U_{p-2}(s)\right) L_{3} u \otimes \psi_{m}(f)\right\|^{2}\right] d s .
\end{aligned}
$$


We now establish (6.4) by induction on $p$, noting that when $p=1$,

$$
\begin{aligned}
& \left\|\left(U_{p}(t)-U_{p-1}(t)\right) u \otimes \psi_{m}(f)\right\|^{2} \\
& \quad=\left\|\int_{0}^{t}\left(d A^{\dagger} \tilde{L}_{1}+\tilde{L}_{2} d A+\tilde{L}_{3} d t\right) u \otimes \psi_{m}(f)\right\|^{2} \\
& \quad=\left\|A^{\dagger}(t) L_{1} u \otimes \psi_{m}(f)-A(t) L_{2} u \otimes \psi_{m}(f)+t L_{3} u \otimes \psi_{m}(f)\right\|^{2} \\
& \quad \leqq 3\left(t+t^{2}\right) C^{2}\|u\|^{2}\left\|\psi_{m}(f)\right\|^{2} \\
& \quad \leqq \exp \left\{t+\int_{0}^{t} \sum_{j=1}^{m}\left|f_{j}\right|^{2}\right\} t C^{2}(2 m+2)^{m}\|u\|^{2} \exp \sum_{j=1}^{m}\left\|f_{j}\right\|^{2}
\end{aligned}
$$

using (2.4), together with the inequality $\left\|A^{\#}(t)\right\|^{2} \leqq t$. Thus (6.4) holds in this case. Making the inductive assumption in (6.6) that

$$
\begin{aligned}
& \left\|\left(U_{p-1}(s)-U_{p-2}(s)\right) L_{j} u \otimes \psi_{m}(f)\right\|^{2} \\
& \leqq((p-1) !)^{-1} \exp \left\{s+\int_{0}^{s} \sum_{j=1}^{m}\left|f_{j}\right|^{2}\right\} s^{p-1} C^{2 p-2}(2 m+2)^{p-1+m} \\
& \cdot\left\|L_{j} u\right\|^{2} \exp \sum_{j=1}^{m}\left\|f_{j}\right\|^{2}
\end{aligned}
$$

and using the inequality

$$
m\left((2 m)^{p+m-1}+(2 m)^{p+m-2}\right)+(2 m+2)^{p-1+m} \leqq(2 m+2)^{p+m}
$$

establishes (6.4) in all generality.

From the estimate (6.4) it is clear that

$$
\begin{aligned}
U(t) u \otimes \psi_{m}(f) & =\lim _{p} U_{p}(t) u \otimes \psi_{m}(f) \\
& =u \otimes \psi_{m}(f)+\sum_{p=1}^{\infty}\left(U_{p}(t)-U_{p-1}(t)\right) u \otimes \psi_{m}(f)
\end{aligned}
$$

exists and defines an even adapted process $U$. Moreover, the convergence is uniform for $t$ in finite intervals enabling us to take strong limits on vectors of form $u \otimes \psi_{m}(f)$ on both sides of (6.2) to conclude that $U$ satisfies (6.1).

Theorem 6.2. The solution $U$ of (6.1) is unique.

Proof. It suffices to show that the only solution of (6.1) with the initial condition replaced by $U(0)=0$ is $U(t) \equiv 0$. Since if $U$ satisfies this equation, so do its even and odd parts and we may assume that $U$ is of definite parity. Then from the estimate (5.1), for arbitrary $u \in h_{0}, m \geqq 0, f_{1}, \ldots, f_{m} \in h$,

$$
\begin{aligned}
& \frac{d}{d t}\left\|U(t) u \otimes \psi_{m}(f)\right\|^{2} \\
& \leqq \\
& \quad \sum_{j=1}^{m}\left\{\left|f_{j}(t)\right|^{2}\left[\left\|U(t) \theta u \otimes \psi_{m-1}\left(f^{j}\right)\right\|^{2}+\left\|U(t) u \otimes \psi_{m}(f)\right\|^{2}\right]\right. \\
& \left.\quad+\left\|U(t) L_{2} \theta u \otimes \psi_{m-1}\left(f^{j}\right)\right\|^{2}\right\} \\
& \quad+\left\|U(t) u \otimes \psi_{m}(f)\right\|^{2}+(m+1)\left\|U(t) L_{1} u \otimes \psi_{m}(f)\right\|^{2}+\left\|U(t) L_{3} u \otimes \psi_{m}(f)\right\|^{2} .
\end{aligned}
$$


We prove that $U(t) u \otimes \psi_{m}(f)=0$ by induction on $m$. When $m=0$ (6.8) becomes $\frac{d}{d t}\left\|U(t) u \otimes \psi_{0}\right\|^{2} \leqq\left\|U(t) u \otimes \psi_{0}(f)\right\|^{2}+\left\|U(t) L_{1} u \otimes \psi_{0}(f)\right\|^{2}+\left\|U(t) L_{3} u \otimes \psi_{0}(f)\right\|^{2}$, whence we obtain, recalling that $U(0)=0$ and using the integrating factor $e^{-t}$,

$$
\left\|U(t) u \otimes \psi_{0}\right\|^{2} \leqq \int_{0}^{t} e^{t-s}\left\{\left\|U(s) L_{1} u \otimes \psi_{0}\right\|^{2}+\left\|U(s) L_{3} u \otimes \psi_{0}\right\|^{2}\right\} d s .
$$

Since $U$ is a stochastic integral, $s \rightarrow U(s) L_{j} u \otimes \psi_{0}$ is continuous and hence bounded on $[0, t]$ for each $u \in h_{0}$. Hence by the principle of uniform boundedness there exists $M_{j}>0$ such that, for all $s \in[0, t]$

$$
\left\|U(s) L_{j} u \otimes \psi_{0}\right\|^{2} \leqq M_{j}\|u\|^{2} .
$$

Iteration of the estimate (6.9) now shows that $\left\|U(t) L_{j} u \otimes \psi_{0}\right\|^{2}=0$. Making the inductive assumption that each $\left\|U(t) v \otimes \psi_{m-1}(g)\right\|=0$, we have from (6.8) that

$$
\begin{aligned}
\frac{d}{d t}\left\|U(t) u \otimes \psi_{m}(f)\right\|^{2} \leqq & \left\{\sum_{j=1}^{m}\left|f_{j}(t)\right|^{2}+1\right\}\left\|U(t) u \otimes \psi_{m}(f)\right\|^{2} \\
& +(m+1)\left\|U(t) L_{1} u \otimes \psi_{m}(f)\right\|^{2}+\left\|U(t) L_{3} u \otimes \psi_{m}(f)\right\|^{2} .
\end{aligned}
$$

Using the integrating factor $\exp \left\{-t-\int_{0}^{t} \sum_{j=1}^{m}\left|f_{j}\right|^{2}\right\}$, we obtain

$$
\begin{aligned}
\left\|U(t) u \otimes \psi_{m}(f)\right\|^{2} \leqq & \int_{0}^{t} \exp \left\{t-s+\int_{s}^{t} \sum_{j=1}^{m}\left|f_{j}\right|^{2}\right\} \\
& \cdot\left\{(m+1)\left\|U(s) L_{1} u \otimes \psi_{m}(f)\right\|^{2}+\left\|U(s) L_{3} u \otimes \psi_{m}(f)\right\|^{2}\right\} d s .
\end{aligned}
$$

A similar argument to that of the case $m=0$ now gives that $\left\|U(t) u \otimes \psi_{m}(f)\right\|^{2}=0$ as required.

We now find necessary conditions on $\left(L_{1}, L_{2}, L_{3}\right)$ for the solution $U$ of (6.1) to be unitary. Since if this is so, $U, U \tilde{L}_{1}, U \tilde{L}_{2}, U \tilde{L}_{3}$ are uniformly bounded on finite intervals, we can use Theorem 5.2 to write

$$
\begin{aligned}
0= & d\left(U^{\dagger} U\right)=d U^{\dagger} \cdot U+U^{\dagger} \cdot d U+d U^{\dagger} \cdot d U \\
= & d A^{\dagger} \tilde{L}_{2}^{\dagger} U^{\dagger} U+\tilde{L}_{1}^{\dagger} U^{\dagger} U d A+\tilde{L}_{3}^{\dagger} U^{\dagger} U d t+d A^{\dagger} U^{\dagger} U \tilde{L}_{1} \\
& +U^{\dagger} U \tilde{L}_{2} d A+U^{\dagger} U \tilde{L}_{3} d t+\tilde{L}_{1}^{\dagger} U^{\dagger} U \tilde{L}_{1} d t
\end{aligned}
$$

Since $U^{\dagger} U=I$ this gives, on equating to zero coefficients of the differentials,

$$
\tilde{L}_{1}^{\dagger}+\tilde{L}_{2}=0, \quad \tilde{L}_{3}^{\dagger}+\tilde{L}_{3}+\tilde{L}_{1}^{\dagger} \tilde{L}_{1}=0 .
$$

Hence for unitarity of $U$ it is necessary that

$$
\left(L_{1}, L_{2}, L_{3}\right)=\left(L,-L^{\dagger}, i H-\frac{1}{2} L^{\dagger} L\right),
$$

where $L \in B\left(h_{0}\right)$ is arbitrary and $H \in B\left(h_{0}\right)$ is self-adjoint. Theorem 6.3 below shows that this condition is also sufficient. 
For a triple of form (6.10) we denote by $\mathscr{L}, \mathscr{L}^{\prime}$ the operators on the Banach space $B\left(h_{0}\right)$,

$$
\begin{aligned}
& \mathscr{L}(X)=i[H, X]-\frac{1}{2}\left(L^{\dagger} L X-2 L^{\dagger} \theta X \theta L+X L^{\dagger} L\right) \\
& \mathscr{L}^{\prime}(X)=-i[H, X]-\frac{1}{2}\left(L^{\dagger} L X-2 L^{\dagger} \theta X \theta L+X L^{\dagger} L\right)
\end{aligned}
$$

Theorem 6.3. A necessary and sufficient condition that the solution $U$ of (6.1) be unitary is that $\left(L_{1}, L_{2}, L_{3}\right)$ be of the form (6.10).

Proof. For the sufficiency we first prove that if (6.10) holds, $U^{\dagger}$ is isometric. From (4.6) we have, for arbitrary $u, v \in h_{0}, m, n \geqq 0, f_{1}, \ldots, f_{m}, g_{1}, \ldots, g_{n} \in h$

$$
\frac{d}{d t}\left\langle U^{\dagger} u \otimes \psi_{m}(f), U^{\dagger} v \otimes \psi_{n}(g)\right\rangle=0
$$

and since $U^{\dagger}(0)=I$ it follows that $U^{\dagger}$ is isometric. To show that $U$ is also isometric we again use (4.6) to write

$$
\begin{aligned}
\frac{d}{d t}< & \left.U(t) u \otimes \psi_{m}(f), U(t) v \otimes \psi_{n}(g)\right\rangle \\
= & \sum_{j=1}^{m}(-1)^{m-j} \overline{f_{j}(t)}\left\{-\left\langle U(t) L^{\dagger} \theta u \otimes \psi_{m-1}\left(f^{j}\right), U(t) v \otimes \psi_{n}(g)\right\rangle\right. \\
& \left.+\left\langle U(t) \theta u \otimes \psi_{m-1}\left(f^{j}\right), U(t) L v \otimes \psi_{n}(g)\right\rangle\right\} \\
& +\sum_{k=1}^{n}(-1)^{n-k}\left\{\left\langle U(t) L u \otimes \psi_{m}(f), U(t) \theta v \otimes \psi_{n-1}\left(g^{k}\right)\right\rangle\right. \\
& \left.-\left\langle U(t) u \otimes \psi_{m}(f), U(t) L^{\dagger} \theta v \otimes \psi_{m-1}\left(g^{k}\right)\right\rangle\right\} g_{k}(t) \\
& +\left\langle U(t)\left(i H-\frac{1}{2} L^{\dagger} L\right) u \otimes \psi_{m}(f), U(t) v \otimes \psi_{n}(g)\right\rangle \\
& +\left\langle U(t) u \otimes \psi_{m}(f), U(t)\left(i H-\frac{1}{2} L^{\dagger} L\right) v \otimes \psi_{n}(g)\right\rangle \\
& +\left\langle U(t) L u \otimes \psi_{m}(f), U(t) L v \otimes \psi_{n}(g)\right\rangle
\end{aligned}
$$

Hence the bounded operators $K_{m, n}(f, g ; t)$ defined on $h_{0}$ by

$$
\left\langle u, K_{m, n}(f, g ; t) v\right\rangle=\left\langle U(t) u \otimes \psi_{m}(f), U(t) v \otimes \psi_{n}(g)\right\rangle
$$

satisfy the system of (weak sense) ordinary differential equations

$$
\begin{aligned}
\frac{d}{d t} K_{m, n}(f, g ; t)= & \theta \sum_{j=1}^{m}(-1)^{m-j} \overline{f_{j}(t)}\left[K_{m-1, n}\left(f^{j}, g ; t\right), L\right] \\
& +\theta \sum_{k=1}^{n}(-1)^{n-k}\left[L^{\dagger}, K_{m, n-1}\left(f, g^{k} ; t\right)\right] g_{k}(t)+\mathscr{L}^{\prime}\left(K_{m, n}(f, g ; t)\right)
\end{aligned}
$$

with the initial condition [which follows from $U(0)=I]$

$$
K_{m, n}(f, g ; 0)=\left\langle\psi_{m}(f), \psi_{n}(g)\right\rangle I
$$


We prove by induction on $N=m+n$ that $K_{m, n}(f, g ; t)$ is equal to its initial value for all $t \geqq 0$. When $N=0$ so that $m=n=0,(6.12)$ becomes

$$
\frac{d}{d t} K_{0,0}=\mathscr{L}^{\prime}\left(K_{0,0}\right)
$$

Since $\mathscr{L}^{\prime}$ is bounded the solution with the given initial condition is unique; since $\mathscr{L}^{\prime}(I)=0$, this solution is $K_{0,0}(t) \equiv K_{0,0}(0)=I$. Making the inductive assumption that $K_{m-1, n}\left(f^{j}, g ; t\right), K_{m, n-1}\left(f, g^{k} ; t\right)$ are identically equal to their initial values given by (6.13), we have from (6.12) that

$$
\frac{d}{d t} K_{m, n}(f, g ; t)=\mathscr{L}^{\prime}\left(K_{m, n}(f, g ; t)\right) \text {. }
$$

Hence by the reasoning of the case $N=0, K_{m, n}(f, g ; t)$ is also identically equal to its initial value (6.13). But then from the definition of $K_{m, n}(f, g ; t)$ we see that $\left\langle U(t) u \otimes \psi_{m}(f), U(t) v \otimes \psi_{n}(g)\right\rangle$ is also identically equal to its initial value $\left\langle u \otimes \psi_{m}(f), v \otimes \psi_{n}(g)\right\rangle$, and so $U(t)$ is isometric as required.

\section{Applications}

Let $U$ be the unitary solution of the stochastic differential equation $d U$ $=\mathrm{dA}^{\dagger} U \tilde{L}-U \tilde{L}^{\dagger} d A+U\left(i \tilde{H}-\frac{1}{2} \tilde{L}^{\dagger} \tilde{L}\right) d t, U(0)=I$, where $L, H \in B\left(h_{0}\right)$ and $H=H^{\dagger}$. For each $X \in B\left(h_{0}\right)$ defines the process $(\tilde{X}(t): t \geqq 0)$ by $\hat{X}(t)=U(t) X \hat{\otimes} I U(t)^{\dagger}$. Applying Theorem 5.2 to the processes $U X \hat{\otimes} I$ and $U^{\dagger}$, if $X$ is of definite parity we find

$$
\begin{aligned}
d \hat{X}= & d A^{\dagger}\left(U L X \hat{\otimes} I U^{\dagger}-(-1)^{\delta(X)} U X L \hat{\otimes} I U^{\dagger}\right) \\
& +\left(-(-1)^{\delta(X)} U L^{\dagger} X \hat{\otimes} I U^{\dagger}+U X L^{\dagger} \otimes I U^{\dagger}\right) d A \\
& +U\left(i[H, X]-\frac{1}{2} L^{\dagger} L X+L^{\dagger} \theta X \theta L-\frac{1}{2} X L^{\dagger} L\right) \hat{\otimes} I U^{\dagger} d t \\
= & d A^{\dagger}\left(L X-(-1)^{\delta(X)} X L\right)^{\wedge}+\left(X L^{\dagger}-\left(-1^{\delta(X)} L^{\dagger} X\right)^{\wedge} d A+\mathscr{L}(X)^{\wedge} d t\right.
\end{aligned}
$$

with $\mathscr{L}$ given by (6.11). When $X$ is a Fermion annihilation operator $a$ satisfying $\left\{a, a^{\dagger}\right\}=I, \hat{X}$ is a Fermion diffusion in the sense of [3].

We define the vacuum conditional expectation $\mathbb{E}_{0}: B(\tilde{\mathscr{H}}) \rightarrow B\left(h_{0}\right)$ by

$$
\left\langle u, \mathbb{E}_{0}[J] v\right\rangle=\left\langle u \otimes \Psi_{0}, J v \otimes \Psi_{0}\right\rangle \quad\left[J \in B(\tilde{\mathscr{H}}), u, v \in h_{0}\right],
$$

and define families of operators $\left(T_{t}: t \geqq 0\right)$ on $h_{0}$ and $\left(\mathscr{T}_{t}: t \geqq 0\right)$ on $B\left(h_{0}\right)$ by

$$
T_{t}=\mathbb{E}_{0}[U(t)], \quad \mathscr{T}_{t}(X)=\mathbb{E}_{0}[\hat{X}(t)] \quad\left[X \in B\left(h_{0}\right)\right] .
$$

Theorem 7.1. a) $\left(T_{t}: t \geqq 0\right)$ is a uniformly continuous contraction semigroup on $h_{0}$ with infinitesimal generator

$$
\left.\frac{d}{d t} T_{t}\right|_{t=0}=i H-\frac{1}{2} L^{\dagger} L
$$

b) $\left(\mathscr{T}_{t}: t \geqq 0\right)$ is a uniformly continuous semigroup of completely positive maps on $B\left(h_{0}\right)$ with infinitesimal generator $\mathscr{L}$ given by $(6.11)$. 
Proof. a) $T_{t}$ is clearly a contraction. By Theorem 4.1 with $m=n=0$, for $u, v \in h_{0}$,

$$
\begin{aligned}
\left\langle u,\left(T_{t}-I\right) v\right\rangle & =\int_{0}^{t}\left\langle u \otimes \psi_{0}, U(s)\left(i H-\frac{1}{2} L^{\dagger} L\right) v \otimes \psi_{0}\right\rangle d s \\
& =\int_{0}^{t}\left\langle u, T_{s}\left(i H-\frac{1}{2} L^{\dagger} L\right) v\right\rangle d s,
\end{aligned}
$$

whence

$$
T_{t}=\exp \left(t\left(i H-\frac{1}{2} L^{\dagger} L\right)\right) \text {. }
$$

b) $\mathscr{T}_{t}$ is completely positive being the composition of an ampliation, a unitary conjugation and a conditional expectation, each of which is completely positive. For $u, v \in h_{0}, X \in B\left(h_{0}\right)$ of definite parity, we have

$$
\begin{aligned}
\left\langle u,\left(\mathscr{T}_{t}(X)-X\right) v\right\rangle & =\left\langle u \otimes \psi_{0},\left(U(t) X \hat{\otimes} I U^{\dagger}(t)-X \hat{\otimes} I\right) v \otimes \psi_{0}\right\rangle \\
& =\left\langle U^{\dagger}(t) u \otimes \psi_{0}, X \hat{\otimes} I U^{\dagger}(t) v \otimes \psi_{0}\right\rangle-\left\langle u \otimes \psi_{0}, X \hat{\otimes} I v \otimes \psi_{0}\right\rangle .
\end{aligned}
$$

Since

$$
d\left(X \hat{\otimes} I U^{\dagger}\right)=-(-1)^{\delta(X)} d A^{\dagger} X L U^{\dagger}+X L^{\dagger} U^{\dagger} d A^{\dagger}-X\left(i H+\frac{1}{2} L^{\dagger} L\right) U^{\dagger} d t,
$$

we have, applying Theorem 4.2 with $m=n=0$ to $U^{\dagger}$ and $X \hat{\otimes} I U^{\dagger}$

$$
\left\langle u,\left(\mathscr{T}_{t}(X)-X\right) v\right\rangle=\int_{0}^{t}\left\langle u, \mathscr{T}_{s}(\mathscr{L}(x)) v\right\rangle d s
$$

with $\mathscr{L}$ given by (6.11), whence b) follows.

As in the Boson case perturbation of these semigroups leads to noncommutative Feynman-Kac formulae, in the case [2] of $\left(T_{t}: t \geqq 0\right)$ of the type of [8] and in particular including a Fermion Feynman-Kac formula of the type of [9], and in the case of $\left(\mathscr{T}_{t}: t \geqq 0\right)$ of the type of [1]. The construction of a Fermion stochastic dilation of an arbitrary uniformly continuous completely positive semigroup similar to that of the Boson case [11] is evidently possible. Details will be published elsewhere.

Both authors acknowledge many useful discussions with K. R. Parthasarathy. We are grateful to the referee for correcting a number of inaccuracies and suggesting several clarifications.

\section{References}

1. Accardi, L.: On the quantum Feynman-Kac formula. Rend. Sem. Mat. Fis. Milano 48, 135-180 (1978)

2. Applebaum, D.B.: Fermion stochastic calculus. Nottingham Ph.D. Thesis, 1984

3. Applebaum, D.B., Hudson, R.L.: Fermion diffusions. J. Math. Phys. 25, 858-861 (1984)

4. Barnett, C., Streater, R.F., Wilde, I.: The Ito-Clifford integral. I. J. Funct. Anal. 48, 172-212 (1982); II. J. London Math. Soc. 27, 373-384 (1983); III. Commun. Math. Phys. 89, 13-17 (1983)

5. Chevalley, C.: The construction of certain important algebras. Pub. Math. Soc. Japan 1958

6. Cockroft, A.M., Hudson, R.L.: Quantum mechanical Wiener processes. J. Multivariate Anal. 7, 107-124 (1977) 
7. Hudson, R.L., Streater, R.F.: Ito's formula is the chain rule with Wick ordering. Phys. Lett. 86 A, 277-279 (1981)

8. Hudson, R.L., Ion, P.D.F., Parthasarathy, K.R.: Time-orthogonal unitary dilations and noncommutative Feynman-Kac formulae. I. Commun. Math. Phys. 83, 261-280 (1982)

9. Hudson, R.L., Ion, P.D.F., Parthasarathy, K.R.: Time-orthogonal unitary dilations and noncommutative Feynman-Kac formulae. II. Publ. Res. Inst. Math. Sci. Kyoto (to appear)

10. Hudson, R.L., Parthasarathy, K.R.: Quantum Ito's formula and stochastic evolutions. Commun. Math. Phys. 93, 301-323 (1984)

11. Hudson, R.L., Parthasarathy, K.R.: Stochastic dilations of uniformly continuous completely positive semigroups. Acta Appl. Math. 2, 353-378 (1984)

12. Segal, I.E.: A noncommutative extension of abstract integration. Ann. Math. 57, 595-596 (1953)

13. Segal, I.E.: Tensor algebras over Hilbert spaces. II. Ann. Math. 63, 160-175 (1956)

Communicated by H. Araki

Received May 7, 1984; in revised form July 23, 1984 